\title{
Effective Capacity for Renewal Service Processes with Applications to HARQ Systems
}

\author{
Zheng Shi, Theodoros A. Tsiftsis, Weiqiang Tan, Guanghua Yang, Shaodan Ma, and Mohamed-Slim Alouini
}

\begin{abstract}
Considering the widespread use of effective capacity in cross-layer design and the extensive existence of renewal service processes in communication networks, this paper thoroughly investigates the effective capacity for renewal processes. Exact expressions of the effective capacity at a given quality of service (QoS) exponent are derived for the renewal processes with either constant or variable reward. The simple expressions reveal meaningful insights, such as the monotonicity and bounds of the effective capacity. The analytical results are then applied to evaluate the cross-layer throughput for diverse hybrid automatic repeat request (HARQ) systems, including fixed-rate HARQ (FR-HARQ, e.g., Type I HARQ, HARQ with chase combining (HARQ-CC) and HARQ with incremental redundancy (HARQIR)), variable-rate HARQ (VR-HARQ) and cross-packet HARQ (XP-HARQ). Furthermore, aiming at maximizing the effective capacity via the optimal rate selection, it is disclosed that VRHARQ and XP-HARQ attain almost the same performance, and both of them perform better than FR-HARQ. In contrast, most of the prior outcomes approximate the effective capacity with the lack of insightful discussions that hampers the optimal design of HARQ systems. Finally, numerical results corroborate the analytical ones.
\end{abstract}

Index Terms-Effective capacity, hybrid automatic repeat request, QoS exponent, renewal process.

\section{INTRODUCTION}

5 $\mathrm{G}$ has been envisioned to offer extremely high data rate (on the order of Gbps) and ultra-reliability (higher than

Manuscript received December 18, 2018; revised April 7, 2019 and May 28, 2019; accepted June 2, 2019. This work was supported in part by the National Key Research and Development Program of China under Grant 2017YFE0120600, in part by National Natural Science Foundation of China under Grants 61801192, 61601524 and 61801132, in part by the Science and Technology Planning Project of Guangdong Province under Grant 2018B010114002, in part by the Macau Science and Technology Development Fund under Grant 091/2015/A3, in part by the Natural Science Foundation of Guangdong Province of China under Grant 2018A030310338, in part by the Project of Educational Commission of Guangdong Province of China under Grant 2017KQNCX155, and in part by the Research Committee of University of Macau under Grant MYRG2018-00156-FST. The associate editor coordinating the review of this paper and approving it for publication was Wei Chen. (Corresponding author: Guanghua Yang.)

Zheng Shi and Theodoros A. Tsiftsis are with the School of Intelligent Systems Science and Engineering, Jinan University, Zhuhai 519070, P. R. China (e-mails: shizheng0124@gmail.com, theodoros.tsiftsis@gmail.com).

Guanghua Yang is with the Institute of Physical Internet, Jinan University, Zhuhai 519070, P. R. China (e-mail: ghyang@jnu.edu.cn).

Weiqiang Tan is with the School of Computer Science and Cyber Engineering, Guangzhou University, Guangzhou 510006, P. R. China (e-mail: wqtan@gzhu.edu.cn).

Shaodan Ma is with the State Key Laboratory of Internet of Things for Smart City and the Dept. of Electrical and Computer Engineering, University of Macau, Macao S.A.R., P. R. China. (e-mail: shaodanma@um.edu.mo)

Mohamed-Slim Alouini is with Computer, Electrical, and Mathematical Science and Engineering (CEMSE) Division, King Abdullah University of Science and Technology, Thuwal 23955-6900, Saudi Arabia (e-mail: slim.alouini@kaust.edu.sa).
99.999\%) [1]. To fulfill these demanding requirements, plenty of existing works prefer to evaluate and devise communication systems from physical-layer perspective. However, the constraints of link-layer quality of service (QoS) were rarely considered in the literature, such as queue length limitation and maximum allowable delay [2]. Unfortunately, physicallayer models cannot capture the characteristics of these QoS requirements, which depends on the queueing behavior of the connection model. Ignoring these QoS limitations grossly overestimates the network performance [3], and many QoSconstrained applications cannot be supported [4]. Therefore, it is imperative to come up with a cross-layer performance metric that combines physical-layer parameters and QoS requirements together.

To address the aforementioned issue, the concept of effective capacity was developed in [2] initially by considering the finite buffer size in practice. This concept has been extensively employed to evaluate the maximum supportable arrival rate at a given QoS exponent, where the QoS exponent affects the statistical QoS constraints, including buffer overflow probability and delay-violation probability. Furthermore, the effective capacity enables us to optimally design cross-layer parameters for various wireless systems subject to statistical QoS constraints [5], [6]. However, most of the relevant literature assumed perfect knowledge of channel state information (CSI) at the transmitter, which is an impractical assumption due to the unpredictable noise, quantization errors, etc. Particularly, in the absence of perfect CSI, the retransmission technique of hybrid automatic repeat request (HARQ) is frequently utilized to strengthen the reception reliability [7], [8]. Although the retransmission of the failed packet can improve the effective capacity [9], it incurs high buffer overflow probability [10]. To make better use of HARQ under various QoS constraints, it is of growing interest to study the effective capacity of HARQ systems. Nonetheless, the introduction of HARQ would make the queueing behaviour of the connection vastly involved, and consequently impedes the analysis of effective capacity given diverse QoS requirements. Instead, in [11], large deviation was adopted to convert the physical-layer throughput of HARQ with incremental redundancy (HARQ-IR) into effective capacity approximately. Bearing this idea in mind, the energydelay tradeoff was investigated for HARQ-IR in [12]. It was shown in the high-power regime that HARQ-IR is more energy-efficient than adaptive modulation and coding (AMC). Moreover, the concept of effective throughput was developed to bypass the complex effective capacity in [3]. In [13], the effective capacity of the cooperative automatic repeat request (ARQ) system was examined by considering delay constraint. 
Jensen's inequality was employed to derive an upper-bound for the effective capacity, with which the superiority of the proposed design is verified when compared to the direct transmission. The energy efficiency was further studied for the cooperative ARQ system [14]. Herein, the law of large number was applied to approximate the effective capacity, which is expressed in terms of the mean and the variance of the service process. Besides, in [15], an accurate approximation of effective capacity under small QoS exponent was obtained on the basis of the cumulants of renewal processes. The approximate expression then enabled the investigation of the energy efficiency of HARQ schemes in the presence of statistical queuing constraints in [16]. Whereas, the concept of the effective capacity in [15] represents the maximum arrival rate that can be supported by HARQ systems. No matter whether the conveyed message can be recovered by receiver or not, every HARQ cycle will be counted as a success. Obviously, it will overestimate the link-layer throughput particularly for high probability of the decoding failures. Therefore, only the goodput of HARQ systems was considered into the formulation of the effective capacity in [17], and the effective capacity was obtained by using the recurrence relation approach. The similar results were further extended to examine the outage effective capacity of the buffer-aided diamond relay systems in [18]. However, in both [17], [18], the analytical results are only applicable to fixed-rate HARQ (FR-HARQ) schemes, wherein the transmission rates remain constant during all HARQ rounds.

Unfortunately so far, the effective capacities of more advanced and complex HARQ schemes, e.g., variable-rate HARQ (VR-HARQ) [19] and cross-packet HARQ (XP-HARQ) [20], have never been investigated due to the challenge of analyzing more complicated service process. Moreover, even if the closed-form expressions have been derived for the effective capacity of the conventional HARQ schemes [17], [18], the complex expressions of the effective capacity provided little insights and it is also difficult to extend the analytical results to the general case. Hence, they will impede the effective cross-layer design of HARQ systems to further enhance the system performance. To combat this issue and generalize the analytical results, we notice that the HARQ transmission model can actually be described by a renewal reward process [21]. Specifically, the event that the transmitter halts HARQ transmissions for the current message is recognized to be a renewal, and the number of the transmitted information bits reflects the reward received from the renewal. In this paper, we first derive a simple exact expression for the effective capacity of the network service that follows constant reward renewal process. The results are further extended to the general renewal process with variable reward. The simple analytical expressions not only offer accurate approximation for the effective capacity under small QoS exponent, but also facilitate the extraction of further meaningful insights. In particular, the effective capacity decreases with the QoS exponent and is bounded. The analytical results are then applied to calculate link-layer throughputs for different HARQ systems, including the conventional FR-HARQ (e.g., Type I HARQ, HARQ with chase combining (HARQ-CC), HARQ-IR), VR-HARQ and XP-HARQ. Numerical examples are finally presented to confirm the proposed approach compared with the already existing ones. Furthermore, aiming to maximize the effective capacity through the optimal rate selection, the numerical results reveal that XP-HARQ and VR-HARQ reach almost the same performance in terms of the optimal effective capacity.

The remainder of this paper is structured as follows. Section II presents preliminaries on effective capacity and HARQ schemes. In Section III, the effective capacity for the renewal process with constant reward is derived. Section IV then extends the results to the general renewal reward process. The analytical results are further applied to evaluate the effective capacity of various HARQ systems in Section V. Numerical results are presented for verifications and discussions in Section VI. Section VII finally concludes this paper. Moreover, Table I provides a list of symbols used in the paper.

\section{Preliminaries}

We first give some useful preliminaries on HARQ schemes, effective capacity and renewal reward process-based modelling, which lay the foundation for the thorough analysis of the effective capacity in various HARQ systems.

\section{A. HARQ Schemes}

As shown in Fig. 1, the delivered message is first encoded into multiple subcodewords according to the HARQ mechanism. The subcodewords will be sent sequentially until the positive acknowledgment (ACK) message is fed back or the maximum number of transmissions is reached. On the basis of whether all the subcodeword lengths are fixed or not, HARQ scheme can be classified into FR-HARQ and VRHARQ. According to various encoding/decoding operations performed at the transceiver, the conventional FR-HARQ can be further categorized into three types, i.e., Type I HARQ, HARQ-CC and HARQ-IR [21]. To be specific, Type I HARQ decodes the message based on the currently received packet. To further improve the reliability, HARQ-CC and HARQIR employ diversity combining and code combining for joint decoding with the erroneously received packets, respectively. Unlike the conventional FR-HARQ, VR-HARQ assumes the variable lengths of the subcodewords for further throughput enhancement [19]. It is worth noting that both FR- and VRHARQ schemes do not include new information bits into retransmissions, they may yield the waste of mutual information. To overcome this shortcoming, VR-HARQ is proposed to add new information bits to retransmissions for possible redundant mutual information [20]. For the simplification of the analysis, the receptions of the HARQ feedback messages are assumed to be error-free and delay-free.

\section{B. Effective Capacity}

The cross-layer model of the typical buffer-limited HARQ system is shown in Fig. 1. The buffer with finite size will lead to the occurrence of buffer overflow, which requires the service arrival rate $\mu$ being properly chosen under certain QoS constraints. Towards this end, the concept of the 
TABLE I: List of Symbols.

\begin{tabular}{l||l}
\hline Symbol & Definition \\
\hline$\mu, Q, \tau, \theta$ & Constant arrival rate, queue length, buffer threshold, QoS exponent, respectively. \\
$C_{e}, C_{e, t}$ & Effective capacities for infinite and finite time $t$, respectively. \\
$S_{t}, N_{t}$ & The total amount of reward received until time $t$, the renewal counting process. \\
$X_{i}, K$ & The interarrival time of the $i$-th renewal, the maximum value of interarrival time. \\
$\mathcal{S}_{n}$ & The total time consumed till the end of the $i$-th renewal. \\
$\mathcal{F}(s), \mathcal{X}(z)$ & Laplace and Z-transforms of the distribution of $X_{i}$, respectively. \\
$q_{k}, q_{k, s}$ & The probability that $X_{i}$ equals to $k$, the probability that the renewal event ends with interval length \\
$\mathfrak{S}_{i}$ & $k$ and termination state $s$. \\
$R$ & The channel/termination state of the $i$-th renewal. \\
$\mathcal{R}\left(X_{i}, \mathfrak{S}_{i}\right)$ & The constant reward. \\
$\mathbf{n}_{t}$ & The reward received from $i$-th renewal and $R_{k, s} \triangleq \mathcal{R}\left(X_{i}=k, \mathfrak{S}_{i}=s\right)$. \\
$\mathcal{K}$ & The vector of renewal counting processes that count the numbers of $R_{k, s}$ s achieved up to time $t$. \\
$b_{k}, L_{k}$ & The maximum number of HARQ transmissions. \\
& The number of the new information bits introduced in the $k$-th transmission, the length of the \\
& $k$-th subcodeword. Besides, $b$ and $L$ denote the number of information bits and the subcodeword \\
$\mathcal{R}, \mathcal{R}_{k}, \breve{\mathcal{R}}$ & length contained in the initial transmission, respectively. \\
\cline { 2 - 2 } & $\mathcal{R}=b / L, \mathcal{R}_{k}=b / L_{k}$ and $\breve{\mathscr{R}}=b_{l} / L$ are defined as the transmission rates. \\
\hline
\end{tabular}

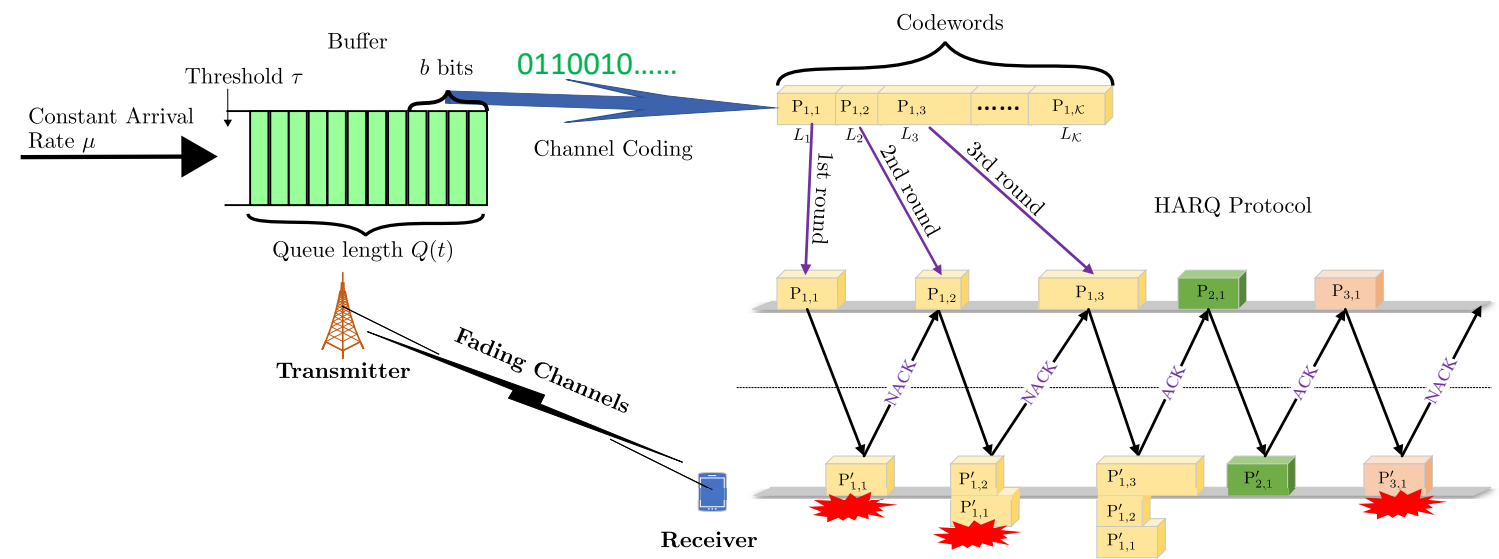

Fig. 1: Cross-layer model for the buffer-limited HARQ System.

effective capacity was developed to specify the maximum supportable arrival rate, i.e., $C_{e}=\max \{\mu\}$ [2]. By assuming a constant arrival rate, buffer overflows will happen if the queue length $Q$ exceeds the buffer threshold $\tau$, and it cannot be avoided under limited buffer size. Based on the theory of large deviations, the buffer overflow probability can be approximated as $\operatorname{Pr}\{Q \geq \tau\} \approx e^{-\theta \tau}$ for large values of $\tau$ [22], where $Q$ and $\tau$ stand for the stationary queue length and buffer's threshold, respectively. It is worth noting that the QoS exponent $\theta$ plays a critical role in connecting the physical and link layers. The QoS exponent $\theta$ implies how fast the buffer overflow probability $\operatorname{Pr}\{Q \geq \tau\}$ decays exponentially with $\tau$. Accordingly, once a constraint is imposed on the buffer overflow probability, the QoS exponent $\theta$ can be determined as $\theta \approx-\ln (\operatorname{Pr}\{Q \geq \tau\}) / \tau$. Assume that the service process satisfies the Gärtner-Ellis theorem [22], given the QoS exponent $\theta$, the effective capacity is explicitly obtained by the limit [2]

$$
C_{e}=-\lim _{t \rightarrow \infty} \frac{1}{\theta t} \ln \mathbb{E}\left\{e^{-\theta S_{t}}\right\},
$$

where $\mathbb{E}\{\cdot\}$ is the expectation operator and $S_{t}$ is the timeaccumulated service process representing the total amount of reward received until time $t$. Moreover, the definition of the effective capacity was further expanded to the case with finite time $t$ in [17], i.e., $C_{e, t}=-\frac{1}{\theta t} \ln \mathbb{E}\left\{e^{-\theta S_{t}}\right\}$.

\section{Renewal Reward Process-Based HARQ Channel Service Modelling}

Note that the cross-layer HARQ system can be modelled by using the renewal reward process [21]. Specifically, the event that HARQ retransmissions stop for the current message is treated as a renewal. Each time when a renewal takes place, we receive a reward that commonly refers to the total number of the information bits carried by the message. Moreover, most of the network services obey renewal reward processes, which incorporate the HARQ channel service as a special case.

Considering whether the number of the delivered information bits in the HARQ scheme is fixed or not, the reward of each HARQ renewal event could be constant or variable. More specifically, the renewal reward of the FR-HARQ scheme is 
kept unchanged because of the same number of information bits transmitted in each HARQ round. However, the outage events usually occur for the truncated HARQ scheme, which has a limited number of transmissions. In the circumstance, the actual reward for an outage renewal event becomes zero. By disregarding the null reward, the cross-layer throughput will be overestimated under the assumption of constant reward. In [17], [18], the outage effective capacity was developed to combat this issue. Moreover, the variable reward of the renewal event must be taken into account for XP-HARQ because of the possible inclusion of new information bits in retransmissions. Hence, HARQ channel services should be thoroughly investigated by using the following two models of the renewal reward process.

1) Renewal Process with Constant Reward-Based Modelling: If each time a renewal occurs with a constant reward $R$, the total amount of the accumulated reward is

$$
S_{t}=R N_{t},
$$

where $N_{t}$ denotes the renewal counting process that counts the number of renewals that occur up until time $t$. More precisely, the counting process $N_{t}$ is defined as $N_{t}=\max \left\{n: \mathcal{S}_{n} \leq t\right\}$, where $\mathcal{S}_{n}=\sum_{i=1}^{n} X_{i}, X_{i}$ is the random time between two consecutive renewals. We assume that interarrival times $\left\{X_{i}, i \in \mathbb{N}\right\}$ are independent and identically distributed (i.i.d.). In the FR-HARQ modelling, $R$ and $N_{t}$ represent the number of information bits in each message and the total number of messages completely delivered till time $t$, respectively. Besides, $X_{i}$ correspond to the total number of HARQ rounds consumed to transmit the $i$-th message.

2) General Renewal Reward Process-Based Modelling: In general, the reward earned by each renewal could be variable apart from constant [23]. In communication systems, the reward of the $i$-th renewal commonly varies with the length of the renewal interval $X_{i}$ and the channel/termination state $\mathfrak{S}_{i}$ of the $i$-th renewal ${ }^{1}$. We denote by $\left(X_{i}, \mathfrak{S}_{i}\right)$ the $i$-th renewal event state, and denote by $\mathcal{R}\left(X_{i}, \mathfrak{S}_{i}\right)$ the reward received from the $i$-th renewal. By favor of the definition of the renewal counting process $N_{t}, S_{t}$ is given by

$$
S_{t}=\sum_{i=1}^{N_{t}} \mathcal{R}\left(X_{i}, \mathfrak{S}_{i}\right) \text {. }
$$

Particularly in HARQ channel service modelling, if the decoding failures are nonnegligible or the cross-packet transmissions are implemented for HARQ systems, $\mathfrak{S}_{i}$ stands for the decoding state whether the $i$-th message is successfully recovered or not. $\mathcal{R}\left(X_{i}, \mathfrak{S}_{i}\right)$ refers to the total number of information bits for the delivery of the $i$-th message, which ends with the total transmission duration $X_{i}$ and decoding state $\mathfrak{S}_{i}$.

To ease possible extension of the present work, the effective capacity is first analyzed for the renewal service processes with constant reward and variable reward in Sections III and IV, respectively. The analytical results are then applied to obtain

\footnotetext{
${ }^{1}$ For example, $\mathfrak{S}_{i}$ represents the outcome whether the receiver succeeds to decode the message after the termination of HARQ. Moreover, $\mathfrak{S}_{i}$ could also be treated as the channel gains if perfect CSI is known at the transmitter, and AMC scheme is adopted.
}

closed-form expressions for the effective capacity of various HARQ schemes.

\section{Effective Capacity of the Renewal Process WITH CONSTANT REWARD}

By putting (2) into (1), the effective capacity of the renewal service process reduces to [15]

$$
C_{e}=-\lim _{t \rightarrow \infty} \frac{1}{\theta t} \ln \mathbb{E}\left\{e^{-\theta R N_{t}}\right\} .
$$

Clearly, $\mathbb{E}\left\{e^{-\theta R N_{t}}\right\}$ is the expectation taken over the renewal counting process $N_{t}$, such that

$$
\mathbb{E}\left\{e^{-\theta R N_{t}}\right\}=\sum_{n=0}^{\infty} e^{-\theta R n} \operatorname{Pr}\left\{N_{t}=n\right\},
$$

and $\operatorname{Pr}\left\{N_{t}=n\right\}$ is given by

$$
\begin{aligned}
\operatorname{Pr}\left\{N_{t}=n\right\} & =\operatorname{Pr}\left\{\mathcal{S}_{n} \leq t\right\}-\operatorname{Pr}\left\{\mathcal{S}_{n+1} \leq t\right\} \\
& =F_{n}(t)-F_{n+1}(t),
\end{aligned}
$$

where $F_{n}(t)$ denotes the cumulative distribution function $(\mathrm{CDF})$ of $\mathcal{S}_{n}$, i.e.,

$$
F_{n}(t)=\operatorname{Pr}\left\{\mathcal{S}_{n} \leq t\right\}=\operatorname{Pr}\left\{\sum_{i=1}^{n} X_{i} \leq t\right\} .
$$

\section{A. Laplace Transform-Based Analysis}

The independence among $X_{i}$ 's motivates us to calculate (7) through Laplace transform. Denote by $\mathcal{F}(s)$ the Laplace transform of the distribution of $X_{i}$, i.e., $\mathcal{F}(s)=\mathbb{E}\left(e^{-s X_{i}}\right)=$ $\int_{0}^{\infty} e^{-s x} f_{X}(x) d x$, where $f_{X}(x)$ denotes the probability density function (PDF) of $X_{i}$. By applying the convolutional property of Laplace transform to (7), it follows that $F_{n}(t)=$ $\mathcal{L}^{-1}\left\{(\mathcal{F}(s))^{n} / s\right\}(t)$, where $\mathcal{L}^{-1}$ stands for the operator of the inverse Laplace transform. By substituting this result into (6), we get $\operatorname{Pr}\left\{N_{t}=n\right\}=\mathcal{L}^{-1}\left\{(\mathcal{F}(s))^{n}(1-\mathcal{F}(s)) / s\right\}(t)$. Putting this result into (5) together with the formula of the sum of a geometric series, we reach

$$
\mathbb{E}\left\{e^{-\theta R N_{t}}\right\}=\mathcal{L}^{-1}\left\{\frac{1-\mathcal{F}(s)}{s\left(1-e^{-\theta R \mathcal{F}(s))}\right.}\right\}(t),
$$

where $\left|e^{-\theta R} \mathcal{F}(s)\right|<1$. By substituting (8) into (4), the evaluation of the effective capacity can thus be enabled. By taking the Poisson service process as an example, $N_{t}$ follows a Poisson distribution with mean $\lambda t, \mathbb{E}\left\{e^{-\theta R N_{t}}\right\}=$ $\sum_{k=0}^{\infty} e^{-\theta R k} e^{-\lambda t}(\lambda t)^{k} / k !=\exp \left(-\lambda t+\lambda t e^{-\theta R}\right)$. On the other hand, the interarrival time is found to be exponentially distributed with mean $1 /(\lambda t)$. Thus, it follows that $\mathcal{F}(s)=\lambda t /(s+\lambda t)$. By using (8), $\mathbb{E}\left\{e^{-\theta R N_{t}}\right\}=$ $\mathcal{L}^{-1}\left\{1 /\left(s+\lambda t-\lambda t e^{-\theta R}\right)\right\}(t)=\exp \left(-\lambda t+\lambda t e^{-\theta R}\right)$, which consequently justifies the analytical result.

Unfortunately, the application of Laplace transform to HARQ systems $\left(F(s)\right.$ is written in the form of $\left.\sum q_{k} e^{-s k}\right)$ usually results in an infinite number of poles in (8), and in most cases no closed form expression exists for the effective capacity. Moreover, the computation of (8) relying on numerical methods entails higher complexity and accuracy due to the calculation of $\exp (-s k)$ for extremely high $s$. These facts hinder the thorough analysis of the effective capacity. However, if the sequence $\left\{X_{i}, i \in \mathbb{N}\right\}$ is a discrete renewal process (e.g., HARQ channel service), Z-transform can be applied to investigate the effective capacity for more insights. 


\section{B. Z-Transform-Based Analysis}

If $X_{1}, X_{2}, \cdots, X_{i}, \cdots$ are i.i.d. discrete and non-negative integer random variables with the probability mass function (pmf) $\operatorname{Pr}\left\{X_{i}=k\right\}=q_{k}, k \in[0, K], \mathrm{Z}$-transform of the distribution of $X_{i}$ is expressed as $\mathcal{X}(z)=\sum_{k=0}^{K} q_{k} z^{-k}$. Similarly, by applying the convolutional property of Ztransform to (7), we have $F_{n}(t)=\mathcal{Z}^{-1}\left\{(\mathcal{X}(z))^{n} z /(z-1)\right\}$. Combining the latter with (6) leads to $\operatorname{Pr}\left\{N_{t}=n\right\}=$ $\mathcal{Z}^{-1}\left\{\frac{z}{z-1}(\mathcal{X}(z))^{n}(1-\mathcal{X}(z))\right\}$. From (5), $\mathbb{E}\left\{e^{-\theta R N_{t}}\right\}$ can be rewritten as

$$
\mathbb{E}\left\{e^{-\theta R N_{t}}\right\}=\frac{1}{2 \pi \mathrm{i}} \oint_{\mathcal{C}} \frac{z(1-\mathcal{X}(z))}{(z-1)\left(1-e^{-\theta R} \mathcal{X}(z)\right)} z^{t-1} \mathrm{~d} z .
$$

where $\left|e^{-\theta R} \mathcal{X}(z)\right|<1$. By using the definition of $\mathcal{X}(z)$, $\mathbb{E}\left\{e^{-\theta R N_{t}}\right\}$ is further expressed as

$$
\mathbb{E}\left\{e^{-\theta R N_{t}}\right\}=\frac{1-e^{\theta R}}{1-q_{0} e^{-\theta R}} \frac{1}{2 \pi \mathrm{i}} \oint_{\mathcal{C}} \frac{\frac{z^{t+K}(1-\mathcal{X}(z))}{z-1}}{\prod_{i \in \Phi}\left(z-z_{i}\right)} \mathrm{d} z,
$$

where $\Phi \triangleq\left\{z_{i}: z_{i}{ }^{K}\left(1-e^{-\theta R} \mathcal{X}\left(z_{i}\right)\right)=0\right\}$. Clearly, $z_{i} \neq 0$ if $q_{K} \neq 0, \Phi$ is therefore equivalent to $\left\{z_{i}: \mathcal{X}\left(z_{i}\right)=e^{\theta R}\right\}$. Furthermore, $0<\left|z_{i}\right|<1$ can be proved by applying triangle inequality to $\mathcal{X}\left(z_{i}\right)=e^{\theta R}$. We assume that $z_{i}$ 's are distinct for analytical tractability. Thus, the cardinality of $\Phi$ is $K, z^{K}\left(1-e^{-\theta R} \mathcal{X}(z)\right)=\left(1-q_{0} e^{-\theta R}\right) \prod_{k=1}^{K}\left(z-z_{k}\right)$ then follows. The integrand in (10) has $K$ distinct poles $z_{i} \in \Phi$, and it is worthwhile to mention that $z=1$ is not a pole because $\lim _{z \rightarrow 1}(1-\mathcal{X}(z)) /(z-1)=$ constant. The following theorem is utilized to derive (10) in a matrix form.

Theorem 1. If $z_{1}, \cdots, z_{K}$ are distinct, the zero points of the function $\phi(z)$ does not coincide with $z_{1}, \cdots, z_{K}$, and $\phi(z)$ has no poles. The inverse Z-transform of $\psi(z) \triangleq$ $\phi(z) / \prod_{k=1}^{K}\left(z-z_{k}\right)$ can be written in a compact form as

$$
\mathcal{Z}^{-1}\{\psi(z)\}(t)=\frac{1}{2 \pi \mathrm{i}} \oint_{\mathcal{C}} \psi(z) z^{t-1} d z=\frac{\operatorname{det} \mathbf{F}}{\operatorname{det} \mathbf{Z}},
$$

where $\mathrm{i}=\sqrt{-1}, \mathcal{Z}^{-1}(\cdot)$ denotes the inverse Z-transform, $\mathcal{C}$ is the contour path of the integration which encircles all of the poles $z_{1}, \cdots, z_{K}, \mathbf{Z}=\left[\left(z_{i}^{j-1}\right)_{i, j}\right]$ is a Vandermonde matrix of size $K \times K$ and $\mathbf{F} \stackrel{\text { I }}{=}$ $\left[\left(z_{i}{ }^{j-1}\right)_{i, 1 \leq j \leq K-1},\left(z_{i}{ }^{t-1} \phi\left(z_{i}\right)\right)_{i, K}\right]$ with size $K \times K$ and det $\cdot$ refers to the determinant operation.

Proof: The proof is omitted here to conserve space, and interested readers are referred to the full version of the paper on arXiv for the details [24].

Accordingly, by using Theorem $1, \mathbb{E}\left\{e^{-\theta R N_{t}}\right\}$ is obtained as

$$
\mathbb{E}\left\{e^{-\theta R N_{t}}\right\}=\frac{1-e^{\theta R}}{1-q_{0} e^{-\theta R}} \frac{\operatorname{det} \mathbf{A}}{\operatorname{det} \mathbf{B}},
$$

where $\mathbf{A}=\left[\left(z_{i}^{j-1}\right)_{i, 1 \leq j \leq K-1},\left(z_{i}{ }^{t+K} /\left(z_{i}-1\right)\right)_{i, K}\right]$ with size $K \times K$ and $\mathbf{B}=\left[\left(z_{i}^{j-1}\right)_{i, j}\right]$ is a Vandermonde matrix of size $K \times K$. Plugging (12) into (4) leads to

$C_{e}=-\lim _{t \rightarrow \infty} \frac{1}{\theta t} \ln \frac{\left(1-e^{\theta R}\right) \operatorname{det} \mathbf{A}}{\left(1-q_{0} e^{-\theta R}\right) \operatorname{det} \mathbf{B}}=-\lim _{t \rightarrow \infty} \frac{1}{\theta t} \ln \operatorname{det} \mathbf{A}$.
Without loss of generality, let $z_{1}$ be the largest in absolute value in $\Phi$, i.e., $\left|z_{1}\right|=\max \left\{\left|z_{i}\right|, i \in[1, K]\right\}$, we have

$$
\begin{aligned}
& C_{e} \\
& =-\lim _{t \rightarrow \infty} \frac{\ln z_{1}^{t+K}}{\theta t}\left|\left(z_{i}{ }^{j-1}\right)_{i, 1 \leq j \leq K-1},\left(\frac{\left(z_{i} / z_{1}\right)^{t+K}}{z_{i}-1}\right)_{i, K}\right| \\
& =\frac{\ln z_{1}{ }^{-1}}{\theta}=\frac{\ln \zeta}{\theta}
\end{aligned}
$$

where $\zeta \triangleq z_{1}{ }^{-1}$. In the following, some discussions are expanded on the basis of (14).

1) Calculation of $\zeta$ : It is clear from (14) that $\zeta \geq 1$, and $\zeta$ satisfies

$$
\sum_{k=0}^{K} q_{k} \zeta^{k}=e^{\theta R}
$$

By applying Jensen's inequality to (15), we have

$$
\sum_{k=0}^{K} q_{k} \zeta^{k} \geq \zeta^{\sum_{k=0}^{K} k q_{k}}=\zeta^{\mathbb{E}(X)} .
$$

Thus, $\zeta \leq e^{\frac{\theta R}{\mathbb{E}(X)}}$ follows. Note $\zeta$ is larger than or equal to 1 , $\zeta$ belongs to $\left[1, e^{\frac{\theta R}{\mathrm{E}(X)}}\right]$. The latter eases the calculation of $\zeta$. More specifically, let us define $g(x) \triangleq \sum_{k=0}^{K} q_{k} x^{k}-e^{\theta R}$. Since $g(x)$ is an increasing function of $x$ if $x>0, \zeta$ is definitely the unique zero point of $g(x)$ within the range $x \in\left[1, e^{\frac{\theta R}{\mathbb{E}(X)}}\right]$. Accordingly, the bisection method can be adopted to calculate $\zeta$.

2) Approximation of $C_{e}$ : If $\theta$ is sufficiently small, the effective capacity can be approximated by $C_{e} \approx R / \mathbb{E}(X)-$ $\theta R^{2} \operatorname{Cov}(X) /\left(2 \mathbb{E}(X)^{3}\right)$ from [15]. This can be further confirmed by using Taylor expansion of (14). To proceed, we define $u(\theta)=\ln \zeta$ and $h(u)=\sum_{k=0}^{K} q_{k} e^{k u}$ such that

$$
h(u(\theta))=e^{\theta R} .
$$

By using Taylor series expansion, $u(\theta)$ can be expanded as

$$
u(\theta)=u(0)+u^{\prime}(0) \theta+\frac{u^{\prime \prime}(0)}{2} \theta^{2}+o\left(\theta^{2}\right),
$$

where $o(\cdot)$ refers to the little-O notation, $u^{\prime}$ and $u^{\prime \prime}$ denote the first and second derivatives of $u$ with respect to (w.r.t.) $\theta$, respectively. $u(0)$ and its derivatives can be determined as follows.

It is readily found from (17) that $u(0)=1$. Taking the first derivative w.r.t. $\theta$ at the both sides of (17) leads to $\frac{d h}{d u} u^{\prime}(\theta)=R e^{\theta R}$. With the definition of $h(u), u^{\prime}(\theta)$ can then be obtained as $u^{\prime}(\theta)=R e^{\theta R} / \sum_{k=0}^{K} q_{k} k e^{k u(\theta)}$. Hence, $u^{\prime}(0)=R / \mathbb{E}(X)$. Similarly, taking the first derivative of $u^{\prime}(\theta)$ w.r.t. $\theta$ gives $u^{\prime \prime}(\theta)$ as

$$
\begin{aligned}
& u^{\prime \prime}(\theta)=R^{2} e^{\theta R}\left(\sum_{k=0}^{K} q_{k} k e^{k u(\theta)}\right)^{-3} \times \\
& \left(\left(\sum_{k=0}^{K} q_{k} k e^{k u(\theta)}\right)^{2}-\sum_{k=0}^{K} q_{k} e^{k u(\theta)} \sum_{k=0}^{K} q_{k} k^{2} e^{k u(\theta)}\right) .
\end{aligned}
$$

Thus, $u^{\prime \prime}(0)=-R^{2} \operatorname{Cov}(X) /(\mathbb{E}(X))^{3}$, where $\operatorname{Cov}(\cdot)$ denotes the covariance operator. Substituting $u(0), u^{\prime}(0)$ and $u^{\prime \prime}(0)$ into (18) together with the definition of $u(\theta)$ arrives 
at $\zeta \approx \exp \left(\theta R / \mathbb{E}(X)-\theta^{2} R^{2} \operatorname{Cov}(X) /\left(2 \mathbb{E}(X)^{3}\right)\right)$. Consequently, putting $\zeta$ into (14) leads to the approximation of $C_{e}$, which coincides with the result in [15].

3) Properties of Effective Capacity: As proved in Appendix A, the effective capacity is found to be a decreasing function of QoS exponent $\theta$. Moreover, $C_{e}$ can be bounded as

$$
\frac{R}{K} \leq C_{e} \leq \min \left\{\frac{R}{K}-\frac{\ln q_{K}}{K \theta}, \frac{R}{\mathbb{E}(X)}\right\},
$$

$\lim _{\theta \rightarrow 0} C_{e}=R / \mathbb{E}(X)$, and $\lim _{\theta \rightarrow \infty} C_{e}=R / K$ if $q_{K}>0$.

4) Extension to the General Case: The result of (14) can be extended to a general case if $\left\{X_{i}\right\}$ is a non-negative continuous renewal process. Specifically, the horizontal axis of the distribution of $X_{i}$ can be partitioned into a number of equal intervals, each with length $\Delta x$. This discretization leads to a new discrete random variable $\tilde{X}_{i}$ with pmf given by $\operatorname{Pr}\left\{\tilde{X}_{i}=k\right\}=\int_{k \Delta x}^{(k+1) \Delta x} f_{X}(x) d x \triangleq q_{k}$. Therefore, a similar approach in Subsection III-B can be adopted to approximate the effective capacity, and the approximation can become more accurate by using a higher-resolution discretization. As $\Delta x \rightarrow 0$, it is readily proved that the exact expression of the effective capacity is

$$
C_{e}=\frac{\ln \zeta}{\theta},
$$

where $\zeta$ is the solution to the following equation

$$
\mathbb{E}\left(\zeta^{X}\right)=e^{\theta R}, \zeta \geq 0 .
$$

$\zeta \in\left[1, e^{\frac{\theta R}{\mathbb{E}(X)}}\right]$, and the proof can be found in Appendix B. Furthermore, it is readily proved that the same properties as shown in Subsections III-B1-III-B3 also apply to the general case except for the bounds of the effective capacity in (20). Nevertheless, (20) is applicable to the discrete renewal service process with non-integer interarrival time, and $K$ stands for the maximum interarrival time herein.

\section{EfFective Capacity of the General Renewal REWARD PROCESS}

Likewise, the effective capacity of the general discrete renewal service processes is derived first, and the analytical results are further extended to that of continuous ones. Note that the renewal depends on the interarrival time and the termination state, we define $R_{k, s} \triangleq \mathcal{R}\left(X_{i}=k, \mathfrak{S}_{i}=s\right)$ for notational convenience, where $k \in[1, K]$ and $s \in\left[1, v_{k}\right]$. Moreover, denote by $q_{k, s}$ the probability that the renewal event is ended with interval length $k$ and termination state $s$, i.e., $q_{k, s} \triangleq \operatorname{Pr}\left\{X_{i}=k, \mathfrak{S}_{i}=s\right\}$. Thus, the probability that the interarrival time is $k$ can be obtained as

$$
\operatorname{Pr}\left\{X_{i}=k\right\}=\sum_{j=1}^{v_{i}} \operatorname{Pr}\left\{X_{i}=k, \mathfrak{S}_{i}=j\right\}=\sum_{j=1}^{v_{i}} q_{k, j} .
$$

Adding up the probabilities $q_{k, s}$ of all the renewal states equals to one, i.e., $\sum_{k=1}^{K} \sum_{s=1}^{v_{k}} q_{k, s}=1$. To facilitate the analysis, we define a vector of renewal counting processes that count the numbers of $R_{k, s}$ 's achieved by the network service up to time $t$ as $\mathbf{n}_{t}=\left(\left(n_{t, k, 1}, \cdots, n_{t, k, v_{k}}\right)_{k=1}^{K}\right)$, where $n_{t, k, s}$ represents the number of the reward $R_{k, s}$ 's earned up until time $t$, and the subscript $t$ is omitted in the sequel for simplicity. Moreover, since the maximum interval between two consecutive renewals is $K, \mathbf{n}_{t}$ should satisfy the following constraint as

$$
[t-K+1]^{+} \leq \sum_{k=1}^{K} \sum_{s=1}^{v_{k}} k n_{k, s} \leq t,
$$

where $[x]^{+}=\max \{0, x\}$ is the projection onto the nonnegative orthant. With the above definition, the total amount of reward given in (3) can be rewritten as

$$
S_{t}=\sum_{k=1}^{K} \sum_{s=1}^{v_{k}} n_{k, s} R_{k, s} .
$$

Therefore, the corresponding effective capacity can be obtained as

$$
C_{e}=-\lim _{t \rightarrow \infty} \frac{1}{\theta t} \ln \underbrace{\mathbb{E}\left\{e^{-\theta \sum_{k=1}^{K} \sum_{s=1}^{v_{k}} n_{k, s} R_{k, s}}\right\}}_{\varphi(t)},
$$

where $\varphi(t)$ is given by (27), shown at the top of the next page. Different from Section III, it is impossible to directly apply Z-transform to derive the moment generating function (mgf) of $S_{t}, \varphi(t)$, due to the complex vector form of $\mathbf{n}_{t}$. To address this challenge, the effective capacity with finite time is obtained by using the multinomial distribution of $\mathbf{n}_{t}$.

\section{A. Effective Capacity with Finite Time}

To proceed, $\operatorname{Pr}\left\{\mathbf{n}_{t}=\left(\left(n_{k, 1}, \cdots, n_{k, v_{k}}\right)_{k=1}^{K}\right)\right\}$ is derived first. To do so, we define $\tau=t-\sum_{k=1}^{K} \sum_{s=1}^{v_{k}} k n_{k, s}$ to show the time of the last renewal passed before time $t$. Clearly, it follows from (24) that $\tau$ is bounded as $0 \leq \tau \leq K$, and the interval of the last renewal should be larger than $\tau$, i.e., $X_{N_{t}+1}>\tau$. According to the law of total probability, $\operatorname{Pr}\left\{\mathbf{n}_{t}=\left(\left(n_{k, 1}, \cdots, n_{k, v_{k}}\right)_{k=1}^{K}\right)\right\}$ is given by (28), shown at the top of the next page, where step (a) holds because of the independence among renewals, and step (b) holds by using (23). More specifically, the vector $\mathbf{n}_{t-\tau}$ follows a multinomial distribution with the pmf given by (29), shown at the top of the next page, where $\left(\begin{array}{c}n \\ n_{1}, \cdots, n_{Q}\end{array}\right)=n ! /\left(n_{1} ! \cdots n_{Q} !\right)$ is the multinomial coefficient and $n=\sum_{q=1}^{Q} n_{q}$.

By substituting (28) and (29) into (27) together with some algebraic manipulations, $\varphi(t)$ is finally rewritten as (30) at the top of the following page. Specifically, if $0 \leq t<K$, (30) becomes

$$
\begin{aligned}
& \varphi(t)=\sum_{\sum_{k=1}^{t} \sum_{s=1}^{v_{k}} k n_{k, s} \in[0, t]} e^{-\theta \sum_{k=1}^{t} \sum_{s=1}^{v_{k}} R_{k, s} n_{k, s}} \\
& \times\left(\begin{array}{c}
\sum_{k=1}^{t} \sum_{s=1}^{v_{k}} n_{k, s} \\
\left(n_{k, 1}, \cdots, n_{k, v_{k}}\right)_{k=1}^{t}
\end{array}\right) \prod_{k=1}^{t} \prod_{s=1}^{v_{k}} q_{k, s}^{n_{k, s}} \\
& \times \sum_{i=t-\sum_{k=1}^{t} \sum_{s=1}^{v_{k}} k n_{k, s}+1}^{K} \sum_{j=1}^{v_{i}} q_{i, j}, 0 \leq t<K,
\end{aligned}
$$

and $\varphi(0)=1$. However, for large $t$, the computation of effective capacity with (30) causes higher complexity overhead. To address this issue, we turn to the Z-transform to derive the effective capacity in a closed-form. 


$$
\begin{aligned}
& \varphi(t)=\sum_{\sum_{k=1}^{K} \sum_{s=1}^{v_{k}} k n_{k, s} \in\left[[t-K+1]^{+}, t\right]} e^{-\theta \sum_{k=1}^{K} \sum_{s=1}^{v_{k}} n_{k, s} R_{k, s}} \operatorname{Pr}\left\{\mathbf{n}_{t}=\left(\left(n_{k, 1}, \cdots, n_{k, v_{k}}\right)_{k=1}^{K}\right)\right\} \\
& \operatorname{Pr}\left\{\mathbf{n}_{t}=\left(\left(n_{k, 1}, \cdots, n_{k, v_{k}}\right)_{k=1}^{K}\right)\right\}=\sum_{i=\tau+1}^{K} \operatorname{Pr}\left\{\mathbf{n}_{t}=\left(\left(n_{k, 1}, \cdots, n_{k, v_{k}}\right)_{k=1}^{K}\right) \mid X_{N_{t}+1}=i\right\} \operatorname{Pr}\left\{X_{N_{t}+1}=i\right\} \\
& \stackrel{(a)}{=} \operatorname{Pr}\left\{\mathbf{n}_{t-\tau}=\left(\left(n_{k, 1}, \cdots, n_{k, v_{k}}\right)_{k=1}^{K}\right)\right\} \sum_{i=\tau+1}^{K} \operatorname{Pr}\left\{X_{N_{t}+1}=i\right\} \\
& \stackrel{(b)}{=} \operatorname{Pr}\left\{\mathbf{n}_{t-\tau}=\left(\left(n_{k, 1}, \cdots, n_{k, v_{k}}\right)_{k=1}^{K}\right)\right\} \sum_{i=\tau+1}^{K} \sum_{j=1}^{v_{i}} q_{i, j} \text {, } \\
& \operatorname{Pr}\left\{\mathbf{n}_{t-\tau}=\left(\left(n_{k, 1}, \cdots, n_{k, v_{k}}\right)_{k=1}^{K}\right)\right\}=\left(\begin{array}{c}
\sum_{k=1}^{K} \sum_{s=1}^{v_{k}} n_{k, s} \\
\left(n_{k, 1}, \cdots, n_{k, v_{k}}\right)_{k=1}^{K}
\end{array}\right) \prod_{k=1}^{K} \prod_{s=1}^{v_{k}} q_{k, s} n_{k, s} \\
& \varphi(t)=\sum_{\sum_{k=1}^{K} \sum_{s=1}^{v_{k}} k n_{k, s} \in\left[[t-K+1]^{+}, t\right]} e^{-\theta \sum_{k=1}^{K} \sum_{s=1}^{v_{k}} R_{k, s} n_{k, s}}\left(\begin{array}{c}
\sum_{k=1}^{K} \sum_{s=1}^{v_{k}} n_{k, s} \\
\left(n_{k, 1}, \cdots, n_{k, v_{k}}\right)_{k=1}^{K}
\end{array}\right) \\
& \times \prod_{k=1}^{K} \prod_{s=1}^{v_{k}} q_{k, s}^{n_{k, s}} \sum_{i=t-\sum_{k=1}^{K} \sum_{s=1}^{v_{k}} k n_{k, s}+1}^{K} \sum_{j=1}^{v_{i}} q_{i, j} .
\end{aligned}
$$

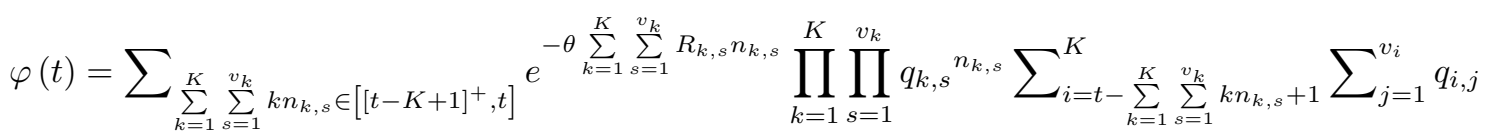

$$
\begin{aligned}
& \times \sum_{\kappa=1}^{K} \sum_{\nu=1}^{v_{\kappa}}\left(\begin{array}{c}
\sum_{k=1}^{K} \sum_{s=1}^{v_{k}} n_{k, s}-1 \\
\left(n_{1,1}, \cdots, n_{1, v_{1}}\right), \cdots,\left(n_{\kappa, 1}, \cdots, n_{\kappa, \nu}-1, \cdots, n_{\kappa, v_{\kappa}}\right), \cdots,\left(n_{K, 1}, \cdots, n_{K, v_{K}}\right)
\end{array}\right) .
\end{aligned}
$$

\section{B. Z-Transform-Based Analysis}

By virtue of the recurrence relation of multinomial coefficient [25, eq.26.4.10], (30) can be rewritten as (32), shown at the top of the page. By assuming $t \geq K$ and switching the order of summations along with some rearrangements, $\varphi(t)$ is represented by a homogeneous difference equation as

$$
\varphi(t)-\sum_{\kappa=1}^{K} a_{\kappa} \varphi(t-\kappa)=0, t \geq K
$$

where $a_{\kappa}=\sum_{j=1}^{v_{\kappa}} q_{\kappa, j} e^{-\theta R_{\kappa, j}}$. As proved in Appendix C, by applying Z-transform to (33), $\varphi(t)$ can be expressed as

$$
\varphi(t)=\sum_{l=1}^{K} \varphi(K-l) \frac{\operatorname{det} \tilde{\mathbf{A}}_{l}}{\operatorname{det} \tilde{\mathbf{B}}}, t \geq K
$$

where $\tilde{\mathbf{A}}_{l}=\left[\left(\tilde{z}_{i}^{j-1}\right)_{i, 1 \leq j \leq K-1},\left(\sum_{\kappa=l}^{K} a_{\kappa} \tilde{z}_{i}^{t+l-\kappa-1}\right)_{i, K}\right]$, $\tilde{\mathbf{B}}=\left[\left(\tilde{z}_{i}^{j-1}\right)_{i, j}\right]$ and $\tilde{z}_{i}$ 's are $K$ distinct roots of the polynomial $z^{K}-\sum_{\kappa=1}^{K} a_{\kappa} z^{K-\kappa}=0$. Without loss of generality, we define $\left|\tilde{z}_{1}\right|=\max \left\{\left|\tilde{z}_{i}\right|, i \in[1, K]\right\}$. Plugging (34) into (26) leads to

$$
\begin{aligned}
C_{e} & =-\lim _{t \rightarrow \infty} \frac{1}{\theta t} \ln \tilde{z}_{1}^{t} \sum_{l=1}^{K} \frac{\varphi(K-l) \tilde{z}_{1}^{l-\kappa-1}}{\operatorname{det} \tilde{\mathbf{B}}} \\
\times & \operatorname{det}\left[\left(\tilde{z}_{i}^{j-1}\right)_{i, 1 \leq j \leq K-1},\left(\sum_{\kappa=l}^{K} a_{\kappa}\left(\frac{\tilde{z}_{i}}{\tilde{z}_{1}}\right)^{t+l-\kappa-1}\right)_{i, K}\right] \\
& =\frac{\ln \tilde{z}_{1}^{-1}}{\theta}=\frac{\ln \tilde{\zeta}}{\theta},
\end{aligned}
$$

where $\tilde{\zeta}=\tilde{z}_{1}^{-1}$. In analogous to Subsection III-B, some discussions are carried out as follows based on (35).

1) Calculation of $\zeta$ : It is obvious from (35) that $\tilde{\zeta}$ is a real number and $\tilde{\zeta} \geq 1$. Similarly, we define $\tilde{g}(x)=\sum_{\kappa=1}^{K} a_{\kappa} x^{\kappa}-$ 1. Hence, $\tilde{\zeta}$ is the zero point of $\tilde{g}(x)$ and uniquely exists owing to its increasing monotonicity within $x \geq 0$. The monotonicity enables us to find the zero point $\tilde{\zeta}$ with bisection method, and $\tilde{\zeta}$ lies in $[1, \exp (\theta \mathbb{E}(\mathcal{R}(X, \mathfrak{S})) / \mathbb{E}(X))]$, where the upper bound is proved in Subsection IV-B3.

2) Approximation of $C_{e}$ : By defining $\tilde{u}(\theta)=\ln \tilde{\zeta}$ and $\tilde{h}(\tilde{u})=\sum_{\kappa=1}^{K} \sum_{j=1}^{v_{\kappa}} q_{\kappa, j} e^{-\theta R_{\kappa, j}} e^{\kappa \tilde{u}}$, we have $\tilde{h}(\tilde{u})=1$. In analogous to Subsection III-B2, $\tilde{u}(\theta)$ can be approximated for small $\theta$ as

$$
\tilde{u}(\theta) \approx \tilde{u}(0)+\tilde{u}^{\prime}(0) \theta+\frac{\tilde{u}^{\prime \prime}(0)}{2} \theta^{2}
$$


where $\tilde{u}^{\prime}$ and $\tilde{u}^{\prime \prime}$ denote the first and second derivatives of $\tilde{u}$ w.r.t. $\theta$, respectively. Clearly, $\tilde{u}(0)=0 . \tilde{u}^{\prime}(\theta)$ and $\tilde{u}^{\prime \prime}(\theta)$ are respectively given by

$$
\tilde{u}^{\prime}(\theta)=\frac{\sum_{\kappa=1}^{K} \sum_{j=1}^{v_{\kappa}} q_{\kappa, j} R_{\kappa, j} e^{-R_{\kappa, j} \theta} e^{\kappa \tilde{u}}}{\sum_{\kappa=1}^{K} \sum_{j=1}^{v_{\kappa}} \kappa q_{\kappa, j} e^{-R_{\kappa, j} \theta} e^{\kappa \tilde{u}}},
$$

and (38) at the top of the next page, respectively. Hence, $\tilde{u}^{\prime}(0)$ and $\tilde{u}^{\prime \prime}(0)$ can be respectively obtained as

$$
\begin{gathered}
\tilde{u}^{\prime}(0)=\frac{\sum_{\kappa=1}^{K} \sum_{j=1}^{v_{\kappa}} q_{\kappa, j} R_{\kappa, j}}{\sum_{\kappa=1}^{K} \kappa \sum_{j=1}^{v_{\kappa}} q_{\kappa, j}}=\frac{\mathbb{E}(\mathcal{R}(X, \mathfrak{S}))}{\mathbb{E}(X)}, \\
\tilde{u}^{\prime \prime}(0)=-\frac{\mathbb{E}(\mathcal{R}(X, \mathfrak{S}) \mathbb{E}(X)-\mathbb{E}(\mathcal{R}(X, \mathfrak{S})) X)^{2}}{(\mathbb{E}(X))^{3}} .
\end{gathered}
$$

(39) implies that $\tilde{u}^{\prime}(0)$ refers to the average reward over time. Particularly for the HARQ schemes, $\tilde{u}^{\prime}(0)$ is the so-called the long term average throughput (LTAT) [21].

Substituting (39) and (40) into (36) along with (35), the effective capacity can be approximated as

$$
\begin{aligned}
C_{e} \approx & \frac{\mathbb{E}(\mathcal{R}(X, \mathfrak{S}))}{\mathbb{E}(X)} \\
& -\frac{\theta \mathbb{E}\left\{(\mathcal{R}(X, \mathfrak{S}) \mathbb{E}(X)-\mathbb{E}(\mathcal{R}(X, \mathfrak{S})) X)^{2}\right\}}{2(\mathbb{E}(X))^{3}} .
\end{aligned}
$$

3) Properties of Effective Capacity: It is proved in Appendix $\mathrm{D}$ that the effective capacity is a decreasing function of $\theta$. Moreover, $C_{e}$ is bounded as

$$
\frac{R_{\hat{\kappa}, \hat{j}}}{K} \leq C_{e} \leq \min \left\{\frac{R_{\hat{\kappa}, \hat{j}}}{\hat{\kappa}}-\frac{\ln q_{\hat{\kappa}, \hat{j}}}{\hat{\kappa} \theta}, \frac{\mathbb{E}(\mathcal{R}(X, \mathfrak{S}))}{\mathbb{E}(X)}\right\},
$$

where $(\hat{\kappa}, \hat{j})=\arg _{(\kappa, j)} \min _{q_{\kappa, j}>0} R_{\kappa, j}$. Accordingly, as $\theta$ approaches to infinity, $C_{e}$ is bounded as

$$
\frac{R_{\hat{\kappa}, \hat{j}}}{K} \leq \lim _{\theta \rightarrow \infty} C_{e} \leq \frac{R_{\hat{\kappa}, \hat{j}}}{\hat{\kappa}} .
$$

Besides, $\lim _{\theta \rightarrow 0} C_{e}=\mathbb{E}(\mathcal{R}(X, \mathfrak{S})) / \mathbb{E}(X)$.

4) Extension to the Continuous Ones: Similarly to Subsection III-B4, the result of (35) can also be extended to the case that $\left\{\left(X_{i}, \mathfrak{S}_{i}\right)\right\}$ is a non-negative continuous renewal process and the reward is variable. Specifically, the horizontal and vertical axis of the distribution of $\left(X_{i}, \mathfrak{S}_{i}\right)$ can be partitioned into a number of equal rectangles, each with area $\Delta x \times \Delta s$. This discretization leads to two new discrete random variables $\left(\tilde{X}_{i}, \tilde{\mathfrak{S}}_{i}\right)$ with pmf given by $\operatorname{Pr}\left\{\tilde{X}_{i}=k, \tilde{\mathfrak{S}}_{i}=\right.$ $l\}=\int_{(k-1) \Delta x}^{k \Delta x} \int_{(l-1) \Delta s}^{l \Delta s} f_{X, \mathfrak{S}}(x, s) d x d s \triangleq q_{k, l}$. Therefore, the similar approach in Subsection IV-B can be employed to approximate the effective capacity. As $\Delta x, \Delta s \rightarrow 0$, we get the exact expression of the effective capacity that is the same as (21), where $\zeta$ is the solution to the following equation

$$
\mathbb{E}_{X, \mathfrak{S}}\left(e^{-\theta \mathcal{R}(X, \mathfrak{S})} \zeta^{X}\right)=1, \zeta \geq 1
$$

Similarly, the effective capacity of the continuous case also follows the same properties as shown in Subsections IV-B1IV-B3 except for the bounds of the effective capacity in (42) and (43). Nevertheless, (42) and (43) are applicable to the discrete renewal service process with non-integer interarrival time, where $K$ is the maximum interarrival time.

\section{ApPlications to HARQ Systems}

\section{A. Fixed-Rate HARQ Systems}

Since HARQ transmissions can be modelled by the renewalreward process [21], the proceeding analysis of the effective capacity can be used to characterize the cross-layer throughput of HARQ [15]. To this end, a renewal of HARQ transmissions is defined as an event that the receiver successfully receives the message or the maximum number of transmissions is reached. It is assumed that the number of transmissions for each message is allowed up to $\mathcal{K}$ due to congestion avoidance.

We assume that each delivered packet contains $b$ information bits, and each one is first encoded into a long codeword. Following the FR-HARQ scheme, the generated codeword is then partitioned into $\mathcal{K}$ subcodewords, and each subcodeword consists of $L$ symbols. Suppose that each symbol duration is normalized to unit. More specifically, $X_{i}$ is the time interval demanded in the successful delivery of the $i$-th message, i.e., $X_{i}=k L$ and $k \leq \mathcal{K}$. The pmf of $X_{i}$, i.e., $q_{k}$ is given by [21]

$$
\operatorname{Pr}\{X=k L\} \triangleq q_{k}=p_{k-1}-p_{k} \mathbb{1}(k \neq \mathcal{K}), k \in[1, \mathcal{K}],
$$

where $p_{k}$ is the outage probability after $k$ HARQ rounds and the indicator function $\mathbb{1}(\mathcal{A})$ is one whenever $\mathcal{A}$ is true and zero otherwise. By applying capacity-achieving codes, the outage expressions for three different HARQ schemes are given respectively by [21]

$$
p_{k}= \begin{cases}\operatorname{Pr}\left\{\log _{2}\left(1+\max \left(\gamma_{1}, \cdots, \gamma_{k}\right)\right) \leq \mathcal{R}\right\}, & \text { Type I } \\ \operatorname{Pr}\left\{\log _{2}\left(1+\sum_{l=1}^{k} \gamma_{l}\right) \leq \mathcal{R}\right\}, & \text { CC } \\ \operatorname{Pr}\left\{\sum_{l=1}^{k} \log _{2}\left(1+\gamma_{l}\right) \leq \mathcal{R}\right\}, & \text { IR. }\end{cases}
$$

where $\mathcal{R}=b / L$ is the transmission rate (normalized reward per renewal), $\gamma_{l}=\gamma_{T} \alpha_{l}$ represents the signal-to-noise ratio (SNR) of the $l$-th transmission, $\gamma_{T}$ and $\alpha_{l}$ correspond to the transmit SNR and the $l$-th channel gain, respectively, and $p_{0}=1$ by convention. The outage performance of HARQ schemes under various fading channels has been extensively investigated. In particular, the outage probabilities of Type I HARQ and HARQ-CC have been derived in closed-form by considering general fading channels in [26, eqs. (8), (11)]. Moreover, the outage expression of HARQ-IR has been given in [27, eq. (17)]. Nonetheless, the specific expressions are omitted here due to space limitation.

1) Maximum Arrival Rate: In [15], the effective capacity is used to assess the maximum arrival rate of HARQ schemes. To do so, by putting (46) into (45) and then combining with (14) and (15), the effective capacities of the three HARQ schemes can be calculated as $C_{e}=\ln \zeta / \theta$, where $\sum_{k=1}^{\mathcal{K}} q_{k} \zeta^{k L}=e^{\theta b}$. By introducing $\bar{\zeta}=\zeta^{L}$ and $\bar{\theta}=L \theta$, the effective capacity can be obtained as

$$
C_{e}=\frac{\ln \bar{\zeta}}{\bar{\theta}}
$$

where $\sum_{k=1}^{\mathcal{K}} q_{k} \bar{\zeta}^{k}=e^{\bar{\theta} \mathcal{R}}$. From (47), the effective capacity of the HARQ scheme is equivalent to that of a new renewal service process with the interarrival time ranged from 1 to $\mathcal{K}$ and the constant reward $\mathcal{R}$. Moreover, $C_{e}$ can be approximated for small $\theta$ by using the Taylor series expansion as illustrated in 


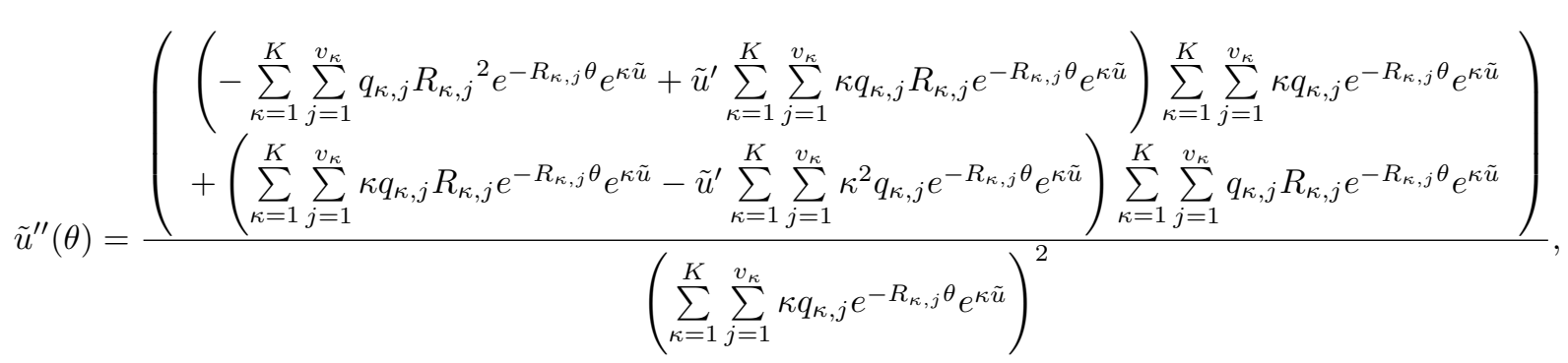

Subsection III-B2. With (20), the effective capacity is bounded as $\mathcal{R} / \mathcal{K} \leq C_{e} \leq \mathcal{R} / \sum_{k=0}^{\mathcal{K}-1} p_{k}$ and $\lim _{\theta \rightarrow \infty} C_{e}=\mathcal{R} / \mathcal{K}$.

Since both the decoding successes and failures are counted as rewards, the effective capacity obtained in (47) indicates the maximum service rate supported by HARQ systems. In addition, the maximum arrival rate can be used to evaluate the throughput of the lossless HARQ scheme, which is allowed to have an infinite number of transmissions, i.e., $\mathcal{K}=\infty$, to guarantee no decoding failures.

2) Outage Effective Capacity: As pointed out in Subsection $\mathrm{V}-\mathrm{A} 1$, the maximum arrival rate does not imply that all the incoming data will be successfully delivered to the receiver because of the presence of decoding failures. In particular, the outage events usually take place for the truncated HARQ schemes, i.e. $\mathcal{K}<\infty$. To address this issue, the outage effective capacity is proposed in [17], [18]. Unlike the maximum arrival rate studied in Subsection $\mathrm{V}-\mathrm{A} 1$, the reward of each renewal is a two-value function for the truncated HARQ schemes. More specifically, the reward is $b$ if the message is successfully decoded and zero otherwise. Therefore, the analytical results in Section IV can be used herein to evaluate the outage effective capacity. For truncated HARQ schemes, $X_{i}$ and $\mathfrak{S}_{i}$ represent the number of HARQ transmissions involved into the delivery of the $i$-th message, and the termination state whether the receiver successfully recovers the message or not, respectively. Hence, the joint pmf $q_{k, s}$ of truncated HARQ schemes is given by

$$
\begin{aligned}
\operatorname{Pr}\{X & =k L, \mathfrak{S}=s\}=q_{k, s} \\
& =\left\{\begin{array}{cc}
p_{k-1}-p_{k}, & k \leq \mathcal{K} \& s=\mathrm{S} \\
p_{\mathcal{K}}, & k=\mathcal{K} \& s=\mathrm{F}
\end{array},\right.
\end{aligned}
$$

where the notations $\mathrm{S}$ and $\mathrm{F}$ denote the success and the failure of the decoding, respectively. The reward function $\mathcal{R}(X, \mathfrak{S})$ is explicitly given by

$$
\mathcal{R}(X=k L, \mathfrak{S}=s)=\left\{\begin{array}{ll}
b, & k \leq \mathcal{K} \& s=\mathrm{S} \\
0, & k=\mathcal{K} \& s=\mathrm{F}
\end{array} .\right.
$$

By using (35), the outage effective capacity can be obtained as $C_{e}^{\text {out }}=\ln \zeta / \theta$, where $\zeta$ satisfies $\sum_{k=1}^{\mathcal{K}} q_{k, \mathrm{~S}} e^{b \theta} \zeta^{k L}+$ $q_{\mathcal{K}, \mathrm{F}} \underline{\zeta}^{\mathcal{K} L}=1$. Similarly, with the same definitions of $\bar{\zeta}=\zeta^{L}$ and $\bar{\theta}=L \theta$, the outage effective capacity can be rewritten as $C_{e}^{\text {out }}=\ln \bar{\zeta} / \bar{\theta}$, where $\bar{\zeta}$ satisfies

$$
\sum_{k=1}^{\mathcal{K}} q_{k, \mathrm{~S}} e^{\mathcal{R} \bar{\theta}} \bar{\zeta}^{k}+q_{\mathcal{K}, \mathrm{F}} \bar{\zeta}^{\mathcal{K}}=1, \bar{\zeta} \geq 0 .
$$

Accordingly, (50) implies that $C_{e}^{\text {out }}$ is equivalent to the outage effective capacity of a simplified HARQ service process with the interarrival time ranged from 1 to $\mathcal{K}$, the QoS exponent $\bar{\theta}$ and the constant reward $\mathcal{R}$. This result is consistent with [17]. By using (41), the outage effective capacity can be approximated for small $\theta$. Moreover, from (42), since $(\mathcal{K} L, \mathrm{~F})=\arg _{(\kappa, j)} \min _{q_{\kappa, j}>0} \mathcal{R}(X, \mathfrak{S})$, the outage effective capacity is bounded as

$$
0 \leq C_{e}^{\text {out }} \leq \min \left\{-\frac{\ln q_{\mathcal{K}, \mathrm{F}}}{\mathcal{K} \bar{\theta}}, \frac{\mathbb{E}(\mathcal{R}(X, \mathfrak{S}))}{\mathbb{E}(X)}\right\},
$$

where the LTAT $\mathbb{E}(\mathcal{R}(X, \mathfrak{S})) / \mathbb{E}(X)$ is given by [28]

$$
\frac{\mathbb{E}(\mathcal{R}(X, \mathfrak{S}))}{\mathbb{E}(X)}=\frac{\mathcal{R}\left(1-p_{\mathcal{K}}\right)}{\sum_{k=0}^{\mathcal{K}-1} p_{k}}
$$

As expected, the outage effective capacity is less than or equal to the LTAT due to the constraint of the limited buffer length. In addition, (43) indicates that

$$
\lim _{\theta \rightarrow \infty} C_{e}^{\text {out }}=0 .
$$

This is different from the maximal arrival rate because erroneously received messages are counted as null rewards. As $\theta$ increases, the buffer overflow probability becomes much more stringent, which consequently leads to the continuous declination of the outage effective capacity.

\section{B. Variable-Rate HARQ-IR Systems}

Unlike the conventional FR-HARQ schemes, the transmission rate of the HARQ-IR scheme could be changeable from one transmission to another. More specifically, we assume that the length of the $k$-th subcodeword is $L_{k}$. By applying capacity achieving channel coding, an outage event happens when the accumulated mutual information is below $b$. The outage probability after $k$ HARQ rounds is thus written as [19]

$$
\hat{p}_{k}=\operatorname{Pr}\left\{\sum_{l=1}^{k} \frac{1}{\mathcal{R}_{l}} \log _{2}\left(1+\gamma_{l}\right)<1\right\},
$$

where $\mathcal{R}_{k}=b / L_{k}$ for $k \in[1, \mathcal{K}]$. From (54), the outage probability of VR-HARQ scheme can be derived in closedform if the fading channels are independently Nakagami-m distributed among different HARQ rounds. The channel gains $\alpha_{1}, \cdots, \alpha_{\mathcal{K}}$ are independent Gamma random variables under the circumstance, and we assume $\alpha_{l} \sim \mathcal{G}\left(m_{l}, \Omega_{l} / m_{l}\right)$, where $m_{l}$ and $\Omega_{l}$ stand for the fading order and average channel power gain, respectively. As proved in the full version of the paper [24, Appendix G], by using Mellin transform, $\hat{p}_{k}$ can be 


$$
\hat{p}_{k}=Y_{1, k+1}^{k, 1}\left[\left(1, \frac{1}{\mathcal{R}_{1}}, \frac{m_{1}}{\gamma_{T} \Omega_{1}}, m_{1}\right), \cdots,\left(1, \frac{1}{\mathcal{R}_{k}}, \frac{m_{k}}{\gamma_{T} \Omega_{k}}, m_{k}\right),(0,1,0,1) \mid 2 \prod_{l=1}^{k}\left(\frac{m_{l}}{\gamma_{T} \Omega_{l}}\right)^{\frac{1}{\mathcal{R}_{l}}}\right],
$$

derived in terms of the generalized Fox's $\mathrm{H}$ function as (55), shown at the top of this page, where the explicit definition of the generalized Fox's $\mathrm{H}$ function $Y_{p, q}^{m, n}(\cdot)$ is omitted here to conserve space (see [29]).

The joint pmf of the interarrival time $\hat{X}$ and the termination state $\hat{\mathfrak{S}}, \hat{q}_{k, s}$, is given by

$$
\begin{aligned}
\operatorname{Pr}\{\hat{X} & \left.=\sum_{l=1}^{k} L_{l}, \hat{\mathfrak{S}}=s\right\}=\hat{q}_{k, s} \\
& =\left\{\begin{array}{cc}
\hat{p}_{k-1}-\hat{p}_{k}, & k \leq \mathcal{K} \& s=\mathrm{S} \\
\hat{p}_{\mathcal{K}}, & k=\mathcal{K} \& s=\mathrm{F}
\end{array} .\right.
\end{aligned}
$$

Moreover, the corresponding reward function $\mathcal{R}(\hat{X}, \hat{\mathfrak{S}})$ is expressed as

$$
\mathcal{R}\left(\hat{X}=\sum_{l=1}^{k} L_{l}, \hat{\mathfrak{S}}=s\right)=\left\{\begin{array}{ll}
b, & k \leq \mathcal{K} \& s=\mathrm{S} \\
0, & k=\mathcal{K} \& s=\mathrm{F}
\end{array} .\right.
$$

Therefore, the effective capacity is obtained as $C_{e}^{\text {out }}=$ $\ln \zeta / \theta$, where $\zeta$ is determined by $\sum_{k=1}^{\mathcal{K}} \hat{q}_{k, \mathrm{~S}} e^{b \theta} \zeta^{\sum_{l=1}^{k} L_{l}}+$ $\hat{q}_{\mathcal{K}, \mathrm{F}} \zeta^{\sum_{l=1}^{\mathcal{K}} L_{l}}=1$. By defining $\hat{\theta}=b \theta$ and $\hat{\zeta}=\zeta^{b}$, the effective capacity can be rewritten as $C_{e}=\ln \hat{\zeta} / \hat{\theta}$, where $\hat{\zeta}$ satisfies

$$
\sum_{k=1}^{\mathcal{K}} \hat{q}_{k, \mathrm{~S}} e^{\hat{\theta}} \hat{\zeta}^{\sum_{l=1}^{k} \mathcal{R}_{l}^{-1}}+\hat{q}_{\mathcal{K}, \mathrm{F}} \hat{\zeta}^{\sum_{l=1}^{\mathcal{K}} \mathcal{R}_{l}^{-1}}=1 .
$$

With (41), the effective capacity can be approximated. In analogous to (51), the effective capacity is bounded as

$$
0 \leq C_{e} \leq \min \left\{-\frac{\ln \hat{q}_{\mathcal{K}, \mathrm{F}}}{\hat{\theta} \sum_{l=1}^{\mathcal{K}} \mathcal{R}^{-1}}, \frac{\mathbb{E}(\mathcal{R}(\hat{X}, \hat{\mathfrak{S}}))}{\mathbb{E}(\hat{X})}\right\}
$$

where the LTAT $\mathbb{E}(\mathcal{R}(\hat{X}, \hat{\mathfrak{S}})) / \mathbb{E}(\hat{X})$ is given by [19]

$$
\frac{\mathbb{E}(\mathcal{R}(\hat{X}, \hat{\mathfrak{S}}))}{\mathbb{E}(\hat{X})}=\frac{1-\hat{p}_{\mathcal{K}}}{\sum_{k=0}^{\mathcal{K}-1} \mathcal{R}_{k+1}{ }^{-1} \hat{p}_{k}} .
$$

Furthermore, the result $\lim _{\theta \rightarrow \infty} C_{e}^{\text {out }}=0$ similar to (53) can be obtained.

\section{Cross-Packet HARQ Systems}

In the retransmissions of both the FR- and VR-HARQ schemes, the retransmitted subcodewords do not involve new information bits. Particularly for FR-HARQ, the provisioning of the throughput close to ergodic capacity is prevented from exploiting the possible redundant mutual information. Instead, the XP-HARQ scheme was devised to avoid the waste of mutual information to substantially improve the throughput [20]. More specifically, the XP-HARQ scheme introduces new information bits in retransmissions besides the redundancy bits. We assume the number of the new introduced information bits in the $k$-th HARQ round is $b_{k}$, and the number of the information bits in the initial transmission is $b_{1}$. During the $k$-th HARQ round, the currently introduced information bits is first concatenated with all the previously introduced ones to construct a long message. The resultant message thus contains $\sum_{l=1}^{k} b_{l}$ information bits, and is then encoded into the $k$-th codeword of length $L$ symbols. According to the decoding conditions of XP-HARQ studied in [20], the outage event takes place after $k$ HARQ rounds if and only if the accumulated mutual information is below the number of the delivered information bits in the current and previous HARQ rounds. Therefore, the outage probability of the XP-HARQ scheme can be obtained as [20]

$$
\begin{aligned}
\breve{p}_{k} & =\operatorname{Pr}\left\{\bigcap_{\kappa=1}^{k}\left(\sum_{l=1}^{\kappa} \operatorname{Llog}_{2}\left(1+\gamma_{l}\right)<\sum_{l=1}^{\kappa} b_{l}\right)\right\} \\
& =\operatorname{Pr}\left\{\bigcap_{\kappa=1}^{k}(\underbrace{\sum_{l=1}^{\kappa} \log _{2}\left(1+\gamma_{l}\right)}_{\mathcal{I}_{\kappa}}<\sum_{l=1}^{\kappa} \breve{R}_{l})\right\},
\end{aligned}
$$

where $\breve{\mathcal{R}_{l}}=b_{l} / L$ and $\bigcap(\cdot)$ represents the intersection of events. Unfortunately, due to the presence of the correlation among the accumulated mutual informations per symbol $\mathcal{I}_{1}, \cdots, \mathcal{I}_{\mathcal{K}}$, it is intractable to derive an exact expression for (61) and there is still no readily available result in the literature. In this paper, $\breve{p}_{k}$ is computed by conducting Monte Carlo simulations.

In order to obtain the effective capacity of the XP-HARQ scheme, the pmf of the interarrival time $\breve{X}$ and the termination state $\breve{\mathfrak{S}}, \breve{q}_{k, s}$, is derived as

$$
\begin{aligned}
\operatorname{Pr}\{\breve{X} & =k L, \breve{S}=s\}=\breve{q}_{k, s} \\
& =\left\{\begin{array}{cc}
\breve{p}_{k-1}-\breve{p}_{k}, & k \leq \mathcal{K} \& s=\mathrm{S} \\
\breve{p}_{\mathcal{K}}, & k=\mathcal{K} \& s=\mathrm{F}
\end{array} .\right.
\end{aligned}
$$

And the reward function $\mathcal{R}(\breve{X}, \breve{S})$ is given by

$$
\mathcal{R}(\breve{X}=k L, \breve{S}=s)=\left\{\begin{array}{cl}
\sum_{l=1}^{k} b_{l}, & k \leq \mathcal{K} \& s=\mathrm{S} \\
0, & k=\mathcal{K} \& s=\mathrm{F}
\end{array}\right.
$$

Accordingly, the effective capacity of XP-HARQ is calculated as $C_{e}^{\text {out }}=\ln \zeta / \theta$, where $\zeta$ is determined by $\sum_{k=1}^{\mathcal{K}} \breve{q}_{k, \mathrm{~S}} e^{\theta \sum_{l=1}^{k} b_{l}} \zeta^{k L}+\breve{q}_{\mathcal{K}, \mathrm{F}} \zeta^{k L}=1$. By defining $\breve{\theta}=L \theta$ and $\breve{\zeta}=\zeta^{L}$, we have $C_{e}=\ln \breve{\zeta} / \breve{\theta}$, where $\breve{\theta}$ is given by

$$
\sum_{k=1}^{\mathcal{K}} \breve{q}_{k, \mathrm{~S}} e^{\breve{\theta} \sum_{l=1}^{k} \breve{R}_{l}} \breve{\zeta}^{k}+\breve{q}_{\mathcal{K}, \mathrm{F}} \breve{\zeta}^{k}=1 .
$$

With (41), the effective capacity can be approximated. In analogous to (51), the effective capacity is bounded as

$$
0 \leq C_{e} \leq \min \left\{-\frac{\ln \breve{q}_{\mathcal{K}, \mathrm{F}}}{\mathcal{K} \breve{\theta}}, \frac{\mathbb{E}(\mathcal{R}(\breve{X}, \breve{\mathfrak{S}}))}{\mathbb{E}(\breve{X})}\right\}
$$


where the LTAT of XP-HARQ, $\mathbb{E}(\mathcal{R}(\breve{X}, \breve{S})) / \mathbb{E}(\breve{X})$, is given by [20]

$$
\frac{\mathbb{E}(\mathcal{R}(\breve{X}, \breve{\mathfrak{S}}))}{\mathbb{E}(\breve{X})}=\frac{\sum_{k=1}^{\mathcal{K}} \breve{\mathcal{R}}_{k}\left(\breve{p}_{k-1}-\breve{p}_{\mathcal{K}}\right)}{\sum_{k=0}^{\mathcal{K}-1} \breve{p}_{k}} .
$$

Furthermore, the similar result to (53) can be demonstrated, i.e., $\lim _{\theta \rightarrow \infty} C_{e}=0$.

Finally, the calculations of the effective capacity for various HARQ schemes are briefly summarized in Table II, as shown at the top of the next page.

\section{VERIFICATIONS AND DiscUSSIONS}

To validate the analysis in Sections III and IV, Monte Carlo simulations are conducted in Fig. 2, which plots the effective capacity $C_{e, t}$ against the time $t$ under two cases. It is worth mentioning that Cases I and II correspond to the renewal processes with constant reward and variable reward, respectively. It can be observed that the results obtained via Laplace and Z-transforms fit well with the simulated ones. In addition, it is found from both cases that the effective capacity with finite time $t, C_{e, t}$, gradually converges to $C_{e}$ as $t$ increases. The correctness of the analytical results is therefore substantiated. Accordingly, numerical examples based on the analytical results of $C_{e}$ are then presented for verifications and discussions. Unless otherwise specified, we set $\gamma_{T}=20 \mathrm{~dB}$, $\theta=10^{-3}, \mathcal{R}=4 \mathrm{bps} / \mathrm{Hz}, \mathcal{K}=5$ and $b=1080 \mathrm{bits}$ in the sequel [30]. In addition, we assume that all HARQ links experience independent Rayleigh fading with unit average power, i.e., $\mathbb{E}\left(\alpha_{l}\right)=\Omega_{l}=1$ and $m_{l}=1$ for $l \in[1, \mathcal{K}]$.

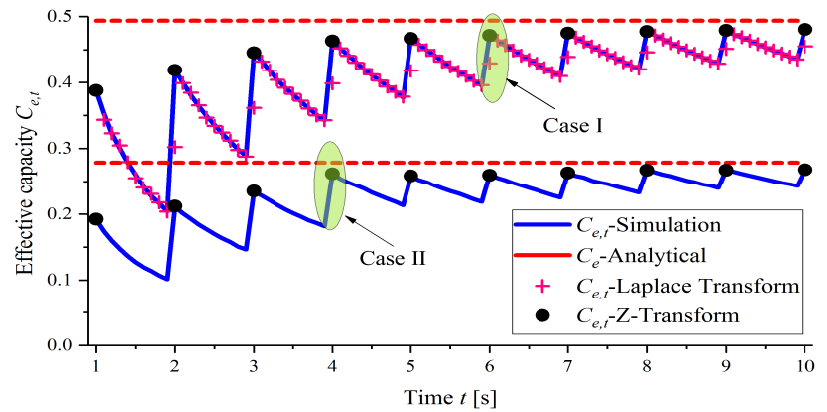

Fig. 2: The effective capacity $C_{e, t}$ versus the time $t$ under two cases with $\theta=0.1$. Case I: $R=1$ and $\left(q_{1}, \cdots, q_{4}\right)=(0.4,0.3,0.2,0.1)$. Case II: $\quad\left(R_{1, \mathrm{~S}}, \cdots, R_{4, \mathrm{~S}}, R_{4, \mathrm{~F}}\right)=(1,1,1,1,0)$ and $\left(q_{1, \mathrm{~S}}, \cdots, q_{4, \mathrm{~S}}, q_{4, \mathrm{~F}}\right)=(0.2,0.2,0.2,0.2,0.2)$, where $\mathrm{S}$ and $\mathrm{F}$ define the two termination states.

\section{A. FR-HARQ Scheme}

To start with, the maximum arrival rate against the QoS exponent $\theta$ for the three conventional FR-HARQ schemes is depicted in Fig. 3, and the approximate results obtained in [15] are provided for comparison. It is observed that $C_{e}$ decreases with $\theta$, which is consistent with our analysis. This is because that higher $\theta$ represents stricter queuing constraints imposed on buffer overflow probability, which limits the increase of service arrival rate, and consequently results in the decrease of the capacity. As shown in Fig. 3, the gap between the approximate and the exact results grows with $\theta$, which justifies the significance of the exact analysis, especially, for large $\theta$. As $\theta$ approaches to infinity, the maximum arrival rate tends to a lower bound that is given by (20), i.e., $\lim _{\theta \rightarrow \infty} C_{e}=$ $R / K=0.8 \mathrm{bps} / \mathrm{Hz}$. Moreover, Fig. 3 further substantiates that HARQ-IR is superior to other HARQ schemes in terms of the maximum arrival rate.

In Fig. 4, the outage effective capacity $C_{e}$ is plotted versus the QoS exponent. It can be seen that the tendency of $C_{e}$ for the three conventional HARQ schemes is the same as Fig. 3, and their approximations given by (41) are also shown for comparison. It is readily found that the approximate results coincide with the exact ones under small QoS exponent. Unlike the maximum arrival rate in Fig. 3, the outage effective capacity approaches to zero as $\theta$ tends to infinity. This difference is due to the fact that the amount of unsuccessfully delivered data is not counted as reward while computing the outage effective capacity. The smaller $\theta$ means the stricter QoS constraint, which needs smaller arrival rate $\mu$ to ensure a tighter constraint on buffer overflow probability.

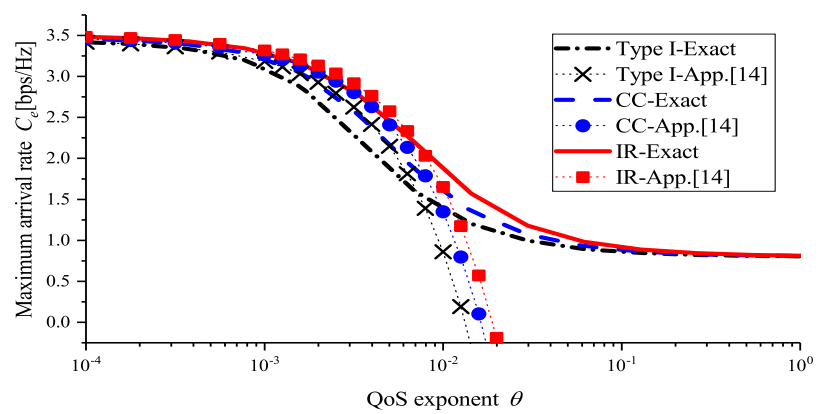

Fig. 3: The maximum arrival rate versus the QoS exponent for FR-HARQ schemes.

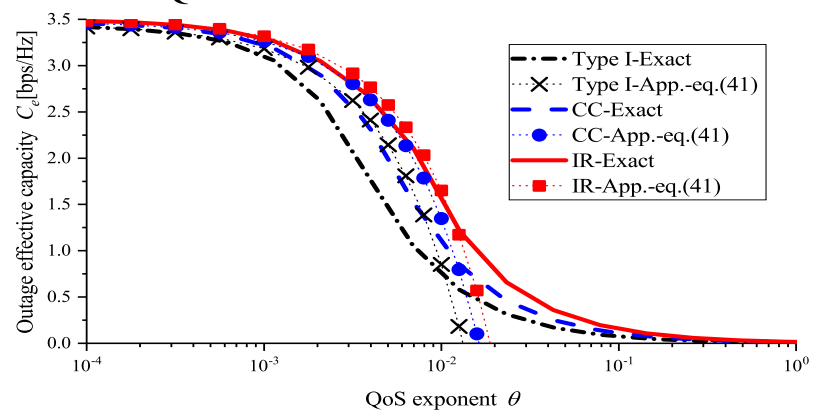

Fig. 4: The outage effective capacity versus the QoS exponent for FR-HARQ schemes.

Fig. 5 illustrates the impact of the transmit SNR $\gamma_{T}$ on the effective capacity, and also shows the comparison between the maximum arrival rate and the outage effective capacity. It is not out of expectation that both the maximum arrival rate and the effective capacity increase with $\gamma_{T}$. Nonetheless, both of them are found to be bounded no matter how high $\gamma_{T}$ is, because the bounds of the maximum arrival rate given in (20) manifest that $0.8 \mathrm{bps} / \mathrm{Hz} \leq C_{e} \leq \mathcal{R} / \mathbb{E}(X)<\mathcal{R}=4 \mathrm{bps} / \mathrm{Hz}$. Whereas, the bounds of the outage effective capacity given by 
TABLE II: The effective capacity for various HARQ schemes.

\begin{tabular}{|c|c|c|c|c|}
\hline $\begin{array}{ll}\text { HARQ } & \text { Metrics } \\
\end{array}$ & $C_{e}$ & $\theta$ & $\zeta$ & $\frac{\mathbb{E}(\mathcal{R}(X, \mathfrak{S}))}{\mathbb{E}(X)}$ \\
\hline $\begin{array}{l}\text { FR-HARQ-Maximum } \\
\text { Arrival Rate }\end{array}$ & \multirow{2}{*}{$C_{e}=\ln \bar{\zeta} / \bar{\theta}$} & \multirow{2}{*}{$\bar{\theta}=L \theta$} & $\sum_{k=1}^{\mathcal{K}} q_{k} \bar{\zeta}^{k}=e^{\bar{\theta} \mathcal{R}}$ & $\frac{\mathcal{R}}{\sum_{k=0}^{\mathcal{K}-1} p_{k}}$ \\
\hline $\begin{array}{l}\text { FR-HARQ-Outage } \\
\text { Effective Capacity }\end{array}$ & & & $\sum_{k=1}^{\mathcal{K}} q_{k, \mathrm{~S}} e^{\mathcal{R} \bar{\theta}} \bar{\zeta}^{k}+q_{\mathcal{K}, \mathrm{F}} \bar{\zeta}^{\mathcal{K}}=1$ & $\frac{\mathcal{R}\left(1-p_{\mathcal{K}}\right)}{\sum_{k=0}^{\mathcal{K}-1} p_{k}}$ \\
\hline VR-HARQ & $C_{e}=\ln \hat{\zeta} / \hat{\theta}$ & $\hat{\theta}=b \theta$ & $\sum_{k=1}^{\mathcal{K}} \hat{q}_{k, \mathrm{~S}} e^{\hat{\theta}} \hat{\zeta}^{\sum_{l=1}^{k} \mathcal{R}_{l}^{-1}}+\hat{q}_{\mathcal{K}, \mathrm{F}} \hat{\zeta}^{\sum_{l=1}^{\mathcal{K}} \mathcal{R}_{l}^{-1}}=1$ & $\frac{1-\hat{p}_{\mathcal{K}}}{\sum_{k=0}^{\mathcal{K}-1} \mathfrak{R}_{k+1}{ }^{-1} \hat{p}_{k}}$ \\
\hline XP-HARQ & $C_{e}=\ln \breve{\zeta} / \breve{\theta}$ & $\breve{\theta}=L \theta$ & $\sum_{k=1}^{\mathcal{K}} \breve{q}_{k, \mathrm{~S}} e^{\breve{\theta} \sum_{l=1}^{k} \breve{R}} \breve{\zeta}^{k}+\breve{q}_{\mathcal{K}, \mathrm{F}} \breve{\zeta}^{k}=1$ & $\frac{\sum_{k=1}^{\mathcal{K}}{\breve{\mathcal{R}_{k}}}_{k}\left(\breve{p}_{k-1}-\breve{p}_{\mathcal{K}}\right)}{\sum_{k=0}^{\mathcal{K}-1} \breve{p}_{k}}$ \\
\hline
\end{tabular}

(42) show that $0 \mathrm{bps} / \mathrm{Hz} \leq C_{e} \leq \mathcal{R}\left(1-p_{\mathcal{K}}\right) / \mathbb{E}(X)<\mathcal{R}=$ $4 \mathrm{bps} / \mathrm{Hz}$.

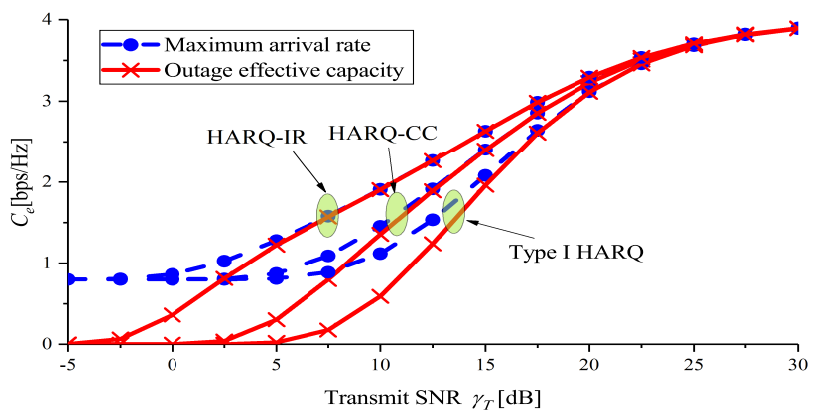

Fig. 5: The comparison between the maximum arrival rate and the outage effective capacity.

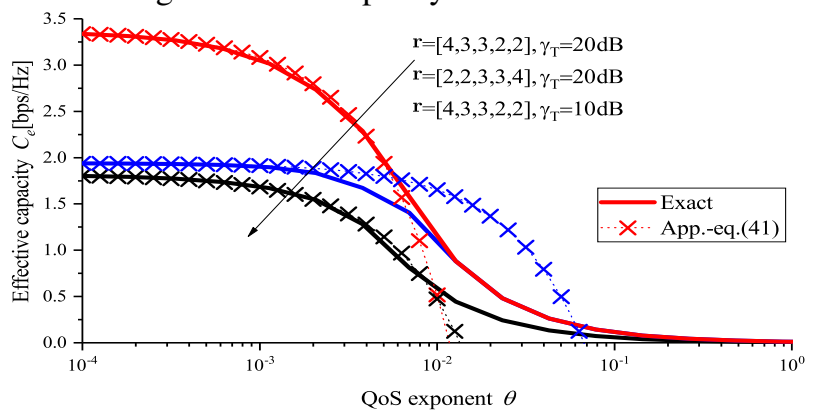

Fig. 6: The effective capacity versus the QoS exponent for the VR-HARQ schemes.

\section{B. VR-HARQ Scheme}

Fig. 6 shows the effective capacity of the VR-HARQ schemes against the effect of the QoS exponent, and the notation $\mathbf{r}$ herein is defined as $\mathbf{r}=\left[\mathcal{R}_{1}, \cdots, \mathcal{R}_{K}\right]$. It is observed in Fig. 6 that the exact results perfectly agree with the approximate ones for small $\theta$. Similarly to Fig. 4, the effective capacity decreases to zero as $\theta$ increases to infinity in Fig. 6. Unsurprisingly, the increase of the transmit SNR improves the effective capacity. For example, for fixed values of $\mathbf{r}=[4,3,3,2,2] \mathrm{bps} / \mathrm{Hz}$ and $\theta=10^{-4}$, the effective capacity increases by $1.5 \mathrm{bps} / \mathrm{Hz}$ if the transmit SNR is increased from $10 \mathrm{~dB}$ to $20 \mathrm{~dB}$. Furthermore, it is seen from this figure that the rate selection has a significant impact on the effective capacity.

\section{XP-HARQ Scheme}

The effective capacity for XP-HARQ scheme is plotted against the QoS exponent in Fig. 7, wherein $\breve{\mathbf{r}}=\left[\breve{R}_{1}, \cdots, \breve{\mathscr{R}}_{K}\right]$. It can be observed that there is a perfect agreement between the exact results and the approximate ones under a small $\theta$. Similarly, the effective capacity of the XP-HARQ scheme is a decreasing function of the QoS exponent as well as the transmit SNR. In addition, it is seen from Fig. 7 that the transmission rates of the XP-HARQ considerably influences the effective capacity.

Note that the selection of transmission rates has a critical impact on the effective capacity, the effective capacity can be maximized via optimizing transmission rates of the HARQ-IR scheme. To compare the three different HARQ-IR schemes, including FR-HARQ-IR, VR-HARQ and XP-HARQ, Fig. 8 exhibits their optimal effective capacities versus the transmit SNR. Nevertheless, the optimal design of transmission rates for HARQ schemes is out of the scope of this paper due to the non-convexity of the objective function. Similarly to [20], we conduct an exhaustive search over the space of following available transmission rates: FR-HARQ-IR and VRHARQ adopt $\mathcal{R}, \mathcal{R}_{k} \in\{1.5,1.75,2, \cdots, 3.75\} \mathrm{bps} / \mathrm{Hz}$, while XP-HARQ adopts $\breve{R}_{1} \in\{1.5,1.75,2, \cdots, 3.75\} \mathrm{bps} / \mathrm{Hz}$ for the initial round and $\breve{\mathscr{R}}_{k} \in\{0,0.25,0.5, \cdots, 3.75\} \mathrm{bps} / \mathrm{Hz}$ for the others. Fig. 8 reveals that VR-HARQ and XP-HARQ can achieve almost the same optimal effective capacity, while FR-HARQ performs the worst. This is because that VRHARQ turns to the variable-length coding to fully exploit the statistical information of fading channels. On the other hand, XP-HARQ attempts to incorporate new information bits into retransmission to make the utmost of the possible redundant mutual information.

\section{CONCLUSIONS}

This paper has derived a unified exact formula for the effective capacity of the renewal network service process at 


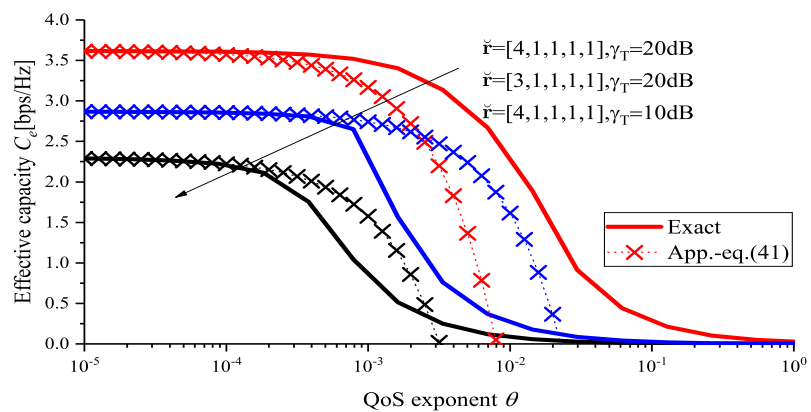

Fig. 7: The effective capacity versus the QoS exponent for the XP-HARQ scheme.

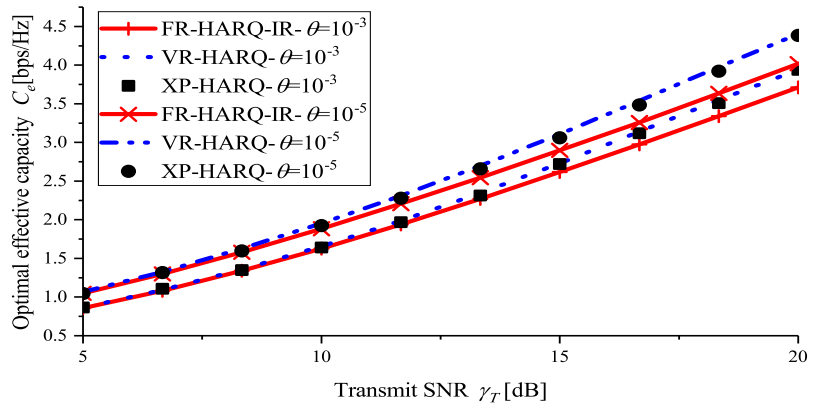

Fig. 8: Optimal effective capacities of different HARQ-IR schemes with parameter $\mathcal{K}=2$.

a given QoS exponent, with which the cross-layer throughput for various HARQ systems has been accurately evaluated, including FR-HARQ (e.g., Type I HARQ, HARQ-CC and HARQ-IR), VR-HARQ and XP-HARQ. The formula not only has gained many insightful results, but also has paved the way for the exact cross-layer design by integrating the channel model of physical-layer to QoS requirements of link-layer. Specifically, the effective capacity has been found to decrease with QoS exponent as well as be bounded. Furthermore, if the transmission rates are optimally chosen to maximize the effective capacity, it has been shown that VR-HARQ and XPHARQ achieve almost the same performance, and both of them surpass FR-HARQ.

It is noticeable that the tractable framework based on the renewal reward process is not limited to evaluate the effective capacity of HARQ schemes, but is believed to have a wide range of applications in wireless communications too. This is due to the fact that numerous communication network services can be modelled by using renewal reward processes, such as cooperative communications, ultra-reliable and low latency communications (URLLC), simultaneous wireless information and power transfer (SWIPT), etc. We plan to extend our analytical approach (e.g., (22) and (44)) to scrutinize the crosslayer performance of these communication systems in our future works.

\section{APPENDIX A}

PROOF OF (20)

By applying the Cauchy-Schwarz inequality to (19), we have $u^{\prime \prime}(\theta) \leq 0$. The following theorem is invoked to demonstrate the decreasing monotonicity of the effective capacity w.r.t. the QoS exponent $\theta$.
Theorem 2. If $f(x)$ is twice differentiable and $f(0)=0$, $\eta(x)=f(x) / x$ is increasing whenever $f^{\prime \prime}(x) \geq 0$ and decreasing otherwise.

Proof: Please refer to the full version of the paper on arXiv for more details [24].

Thanks to the monotonic decreasing function of $C_{e}, C_{e}$ is bounded as $\lim _{\theta \rightarrow \infty} u(\theta) / \theta \leq C_{e} \leq \lim _{\theta \rightarrow 0} u(\theta) / \theta$. Clearly, the corresponding upper bound is $\lim _{\theta \rightarrow 0} u(\theta) / \theta=$ $u^{\prime}(0)=R / \mathbb{E}(X)$. With regard to the lower bound, (17) indicates $e^{K \theta C_{e}} \geq e^{\theta R} \geq q_{K} e^{K \theta C_{e}}$. Accordingly, we have $R / K \leq C_{e} \leq R / K-\ln q_{K} /(K \theta)$. As $\theta$ approaches to infinity, using squeeze theorem yields $\lim _{\theta \rightarrow \infty} u(\theta) / \theta=R / K$ if $q_{K}>0$. Moreover, it is easily proved that $\lim _{\theta \rightarrow 0} C_{e}=$ $u^{\prime}(0)=R / \mathbb{E}(X)$. The bounds of the effective capacity are consequently simplified into (20).

\section{APPENDIX B \\ PROOF OF (22)}

Since $\mathbb{E}\left(\zeta^{X}\right)$ can be approximated as

$$
\begin{aligned}
\mathbb{E}\left(\zeta^{X}\right) \approx \sum_{k=0}^{K} q_{k} \zeta^{k \Delta x} & =\sum_{k=0}^{K} q_{k}\left(\zeta^{-\Delta x}\right)^{-k} \\
& =\mathcal{X}_{\tilde{X}_{i}}(z),
\end{aligned}
$$

where $z=\zeta^{-\Delta x}$ and the approximation becomes an equality as $\Delta \rightarrow 0$, the effective capacity of a renewal process by taking $\tilde{X}_{i}$ as the $i$-th interarrival time can be used to approximate $C_{e}$. Specifically, $C_{e}$ can be approximately obtained by using the same approach in Section III-B as

$$
\begin{aligned}
C_{e} & \approx-\lim _{t \rightarrow \infty} \frac{1}{\theta t} \ln \mathbb{E}\left\{e^{-\theta R N_{t / \Delta x}}\right\} \\
& =\lim _{t \rightarrow \infty} \frac{\left(\frac{t}{\Delta x}+K\right) \ln z^{-1}}{\theta t},
\end{aligned}
$$

where $\sum_{k=0}^{K} q_{k} z^{-k}=e^{\theta R}$, i.e., $\mathcal{X}_{\tilde{X}_{i}}(z)=e^{\theta R}$. Due to the relationship between $\zeta$ and $z$, we have

$$
C_{e}=\lim _{t \rightarrow \infty} \frac{\left(\frac{t}{\Delta x}+K\right) \ln \zeta^{\Delta x}}{\theta_{1} t}=\frac{\ln \zeta}{\theta},
$$

where $\mathbb{E}\left(\zeta^{X}\right)=e^{\theta R}$.

\section{ApPENDIX C \\ PROOF OF (34)}

The solution to the homogeneous difference equation (33) can be solved by employing Z-transform. To this end, it is indispensable to know the initial conditions of the difference equation, and the initial conditions of the $K$-th order linear difference equation are $\varphi(0), \cdots, \varphi(K-1)$. In order to apply Z-transform, we define a new sequence $\varphi_{K}(t)$ by shifting the sequence $\varphi(t)$ to the left by $K$ such that $\varphi_{K}(t)=\varphi(t+K)$, where $t \geq 0$. Hence, (33) can be rewritten in terms of $\varphi_{K}(t)$ as

$$
\varphi_{K}(t)-\sum_{\kappa=1}^{K} a_{\kappa} \varphi_{K}(t-\kappa)=0, t \geq 0,
$$

where $\varphi_{K}(-k)=\varphi(K-k)$ and $k \in[0, K-1]$. Applying the translation property of Z-transform [31, eq.12.4.1] to the 
both sides of (70) gives

$$
\begin{aligned}
& \mathcal{Z}\left\{\varphi_{K}(t)\right\}(z)- \\
& \sum_{\kappa=1}^{K} a_{\kappa} z^{-\kappa}\left(\mathcal{Z}\left\{\varphi_{K}(t)\right\}(z)+\sum_{l=-\kappa}^{-1} \varphi_{K}(l) z^{-l}\right)=0,
\end{aligned}
$$

where $\mathcal{Z}\left\{\varphi_{K}(t)\right\}(z)$ stands for the Z-transform of $\varphi_{K}(t)$. Accordingly, $\mathcal{Z}\left\{\varphi_{K}(t)\right\}(z)$ is simplified as

$$
\mathcal{Z}\left\{\varphi_{K}(t)\right\}(z)=\frac{\sum_{\kappa=1}^{K} a_{\kappa} z^{-\kappa} \sum_{l=1}^{\kappa} \varphi(K-l) z^{l}}{1-\sum_{\kappa=1}^{K} a_{\kappa} z^{-\kappa}} .
$$

By applying the inverse Z-transform to (72), we have

$$
\varphi_{K}(t)=\frac{1}{2 \pi \mathrm{i}} \oint_{c} \mathcal{Z}\left\{\varphi_{K}(t)\right\}(z) z^{t-1} \mathrm{~d} z .
$$

Thus, $\varphi(t)$ can be obtained as

$$
\varphi(t)=\frac{1}{2 \pi \mathrm{i}} \oint_{c} \frac{\sum_{\kappa=1}^{K} \sum_{l=1}^{\kappa} a_{\kappa} \varphi(K-l) z^{t+l-\kappa-1}}{z^{K}-\sum_{\kappa=1}^{K} a_{\kappa} z^{K-\kappa}} \mathrm{d} z, t \geq K .
$$

With the definition of $\tilde{z}_{1}, \cdots, \tilde{z}_{K}$, it follows that $z^{K}-$ $\sum_{\kappa=1}^{K} a_{\kappa} z^{K-\kappa}=\prod_{\kappa=1}^{K}\left(z-\tilde{z}_{\kappa}\right)$. By putting the latter into (74) and using Theorem 1, (74) can be further rewritten as (75), shown at the top of the next page.

\section{APPENDIX D}

\section{Proof of the Properties of EfFEctive Capacity}

\section{A. Decreasing Monotonicity of $C_{e}$ w.r.t. $\theta$}

By defining $\ell_{t}(\theta)=-\ln \mathbb{E}\left\{e^{-\theta S_{t}}\right\} / t$, the effective capacity can be expressed as $C_{e}=\lim _{t \rightarrow \infty} \ell_{t}(\theta) / \theta$. More specifically, $\ell_{t}(0)=0$ and the second derivative of $\ell_{t}(\theta)$ w.r.t. $\theta$ is less than or equal to zero because

$$
\begin{aligned}
\ell_{t}^{\prime \prime}(\theta) & =-\frac{\mathbb{E}\left\{S_{t}^{2} e^{-\theta S_{t}}\right\} \mathbb{E}\left\{e^{-\theta S_{t}}\right\}-\left(\mathbb{E}\left\{S_{t} e^{-\theta S_{t}}\right\}\right)^{2}}{\left(\mathbb{E}\left\{e^{-\theta S_{t}}\right\}\right)^{2} t} \\
& \leq 0,
\end{aligned}
$$

where the last step holds by using Cauchy-Schwarz inequality. Hence, we get $\lim _{t \rightarrow \infty} \ell_{t}(0)=0$ and $\lim _{t \rightarrow \infty} \ell_{t}^{\prime \prime}(\theta) \geq 0$. By using Theorem 2, the decreasing monotonicity of $C_{e}$ w.r.t. $\theta$ is thus proved.

\section{B. Bounds of the Effective Capacity}

Owing to the decreasing monotonicity of the effective capacity, the effective capacity is upper bounded as $C_{e} \leq$ $\lim _{\theta \rightarrow 0} C_{e}$, where the upper bound is given by

$$
\lim _{\theta \rightarrow 0} C_{e}=\lim _{\theta \rightarrow 0} \frac{\tilde{u}(\theta)}{\theta}=\tilde{u}^{\prime}(0)=\frac{\mathbb{E}(\mathcal{R}(X, \mathfrak{S}))}{\mathbb{E}(X)} .
$$

On the other hand, we first define the lowest reward as $R_{\hat{\kappa}, \hat{j}}$, where $(\hat{\kappa}, \hat{j})=\arg _{(\kappa, j)} \min _{q_{\kappa, j}>0} R_{\kappa, j}$. Hence, the effective capacity satisfies

$$
\sum_{\kappa=1}^{K} \sum_{j=1}^{v_{\kappa}} q_{\kappa, j} e^{-\theta\left(R_{\kappa, j}-R_{\hat{\kappa}, \hat{j}}\right)} e^{\kappa \theta C_{e}}=e^{\theta R_{\hat{\kappa}, \hat{j}}} .
$$

From (78), it follows that

$$
\begin{aligned}
q_{\hat{\kappa}, \hat{j}} e^{\hat{\kappa} \theta C_{e}} & \leq \sum_{\kappa=1}^{K} \sum_{j=1}^{v_{\kappa}} q_{\kappa, j} e^{-\theta\left(R_{\kappa, j}-R_{\hat{\kappa}, \hat{j}}\right)} e^{\kappa \theta C_{e}} \\
& \leq e^{K \theta C_{e}} .
\end{aligned}
$$

Thus, $C_{e}$ is bounded as

$$
\frac{R_{\hat{\kappa}, \hat{j}}}{K} \leq C_{e} \leq \frac{R_{\hat{\kappa}, \hat{j}}}{\hat{\kappa}}-\frac{\ln q_{\hat{\kappa}, \hat{j}}}{\hat{\kappa} \theta} .
$$

Combining (77) and (80) leads to (42). Consequently, we complete the proof.

\section{REFERENCES}

[1] P. Popovski, "Ultra-reliable communication in 5G wireless systems," in Proc. International Conference on $5 G$ for Ubiquitous Connectivity (5GU'14), Sep. 2014, pp. 146-151.

[2] D. Wu and R. Negi, "Effective capacity: a wireless link model for support of quality of service," IEEE Trans. Wireless Commun., vol. 2, no. 4, pp. 630-643, Jul. 2003.

[3] D. To, H. X. Nguyen, Q.-T. Vien, and L.-K. Huang, "Power allocation for HARQ-IR systems under QoS constraints and limited feedback," IEEE Trans. Wireless Commun., vol. 14, no. 3, pp. 1581-1594, Mar. 2015.

[4] K. T. Phan, T. Le-Ngoc, and L. B. Le, "Optimal resource allocation for buffer-aided relaying with statistical QoS constraint," IEEE Trans. Commun., vol. 64, no. 3, pp. 959-972, Mar. 2016.

[5] T. Abrão, S. Yang, L. D. H. Sampaio, P. J. E. Jeszensky, and L. Hanzo, "Achieving maximum effective capacity in OFDMA networks operating under statistical delay guarantee," IEEE Access, vol. 5, pp. 1433314346, Aug. 2017.

[6] Y. Hu, M. Ozmen, M. C. Gursoy, and A. Schmeink, "Optimal power allocation for QoS-constrained downlink multi-user networks in the finite blocklength regime," IEEE Trans. Wireless Commun., vol. 17, no. 9, pp. 5827-5840, Sep. 2018.

[7] M. Amjad, L. Musavian, and M. H. Rehmani, "Effective capacity in wireless networks: A comprehensive survey," arXiv preprint arXiv:1811.03681, 2018.

[8] H. Wang, R. Song, and S.-H. Leung, "Throughput analysis of interference alignment for a general centralized limited feedback model," IEEE Trans. Veh. Technol., vol. 65, no. 10, pp. 8775-8781, Oct. 2015.

[9] A. H. Anwar, K. G. Seddik, T. ElBatt, and A. H. Zahran, "Effective capacity of delay-constrained cognitive radio links exploiting primary feedback," IEEE Trans. Veh. Technol., vol. 65, no. 9, pp. 7334-7348, Sep. 2016.

[10] J. Choi, "An effective capacity-based approach to multi-channel lowlatency wireless communications," IEEE Trans. Commun., vol. 67, no. 3, pp. 2476-2486, Mar. 2019.

[11] fading channels," IEEE Commun. Lett., vol. 16, no. 6, pp. 913-916, Jun. 2012.

[12] J. Choi and J. Ha, "On the energy efficiency of AMC and HARQ-IR with QoS constraints," IEEE Trans. Veh. Technol., vol. 62, no. 7, pp. 3261-3270, Sep. 2013.

[13] J. S. Harsini and M. Zorzi, "Effective capacity for multi-rate relay channels with delay constraint exploiting adaptive cooperative diversity," IEEE Trans. Wireless Commun., vol. 11, no. 9, pp. 3136-3147, Sep. 2012.

[14] Y. Hu, J. Gross, and A. Schmeink, "QoS-constrained energy efficiency of cooperative ARQ in multiple DF relay systems," IEEE Trans. Veh. Technol., vol. 65, no. 2, pp. 848-859, Feb. 2016.

[15] Y. Li, M. C. Gursoy, and S. Velipasalar, "On the throughput of hybridARQ under statistical queuing constraints." IEEE Trans. Veh. Technol., vol. 64, no. 6, pp. 2725-2732, Jul. 2015.

[16] Y. Li, G. Ozcan, M. C. Gursoy, and S. Velipasalar, "Energy efficiency of hybrid-ARQ under statistical queuing constraints," IEEE Trans. Commun., vol. 64, no. 10, pp. 4253-4267, Oct. 2016.

[17] P. Larsson, J. Gross, H. Al-Zubaidy, L. K. Rasmussen, and M. Skoglund, "Effective capacity of retransmission schemes: a recurrence relation approach," IEEE Trans. Commun., vol. 64, no. 11, pp. 4817-4835, Nov. 2016.

[18] D. Qiao, "Outage effective capacity of buffer-aided diamond relay systems using HARQ-IR," IEEE Trans. Veh. Technol., vol. 68, no. 1, pp. 540-553, Jan. 2018. 


$$
\begin{aligned}
\varphi(t) & =\frac{\operatorname{det}\left[\left(\tilde{z}_{i}^{j-1}\right)_{i, 1 \leq j \leq K-1},\left(\sum_{\kappa=1}^{K} \sum_{l=1}^{\kappa} a_{\kappa} \varphi(K-l) \tilde{z}_{i}^{t+l-\kappa-1}\right)_{i, K}\right]}{\operatorname{det} \tilde{\mathbf{B}}} \overbrace{\left.\left[\tilde{\mathbf{A}}_{l=1}^{K} \frac{\varphi(K-l)}{\operatorname{det} \tilde{\mathbf{B}}} \operatorname{det} \tilde{z}_{i}^{j-1}\right)_{i, 1 \leq j \leq K-1},\left(\sum_{\kappa=l}^{K} a_{\kappa} \tilde{z}_{i}^{t+l-\kappa-1}\right)_{i, K}\right]}^{\left.\tilde{\mathbf{A}}_{l, k}\right]} .
\end{aligned}
$$

[19] L. Szczecinski, S. R. Khosravirad, P. Duhamel, and M. Rahman, "Rate allocation and adaptation for incremental redundancy truncated HARQ," IEEE Trans. Commun., vol. 61, no. 6, pp. 2580-2590, Jun. 2013.

[20] M. Jabi, A. Benyouss, M. Le Treust, E. Pierre-Doray, and L. Szczecinski, "Adaptive cross-packet HARQ," IEEE Trans. Commun., vol. 65, no. 5, pp. 2022-2035, May 2017.

[21] G. Caire and D. Tuninetti, "The throughput of hybrid-ARQ protocols for the Gaussian collision channel," IEEE Trans. Inf. Theory, vol. 47, no. 5, pp. 1971-1988, Jul. 2001.

[22] C.-S. Chang and J. A. Thomas, "Effective bandwidth in high-speed digital networks," IEEE J. Sel. Areas Commun., vol. 13, no. 6, pp. 10911100, Aug. 1995.

[23] S. M. Ross, Introduction to probability models. Academic press, 2014.

[24] Z. Shi, T. Tsiftsis, W. Tan, G. Yang, S. Ma, and M.-S. Alouini, "Effective capacity for renewal service processes with applications to HARQ systems," arXiv, pp. 1-15, 2018. [Online]. Available: https://arxiv.org/abs/1812.06470

[25] F. W. Olver, NIST handbook of mathematical functions. Cambridge University Press, 2010.

[26] Z. Shi, S. Ma, F. Hou, K.-W. Tam, and Y.-C. Wu, "Optimal power allocation for HARQ schemes over time-correlated Nakagami-m fading channels," in Proc. IEEE Int. Conf. on Commun. Syst. (ICCS'16), Jan. 2016, pp. $1-6$.

[27] Z. Shi, S. Ma, G. Yang, K. W. Tam, and M. Xia, "Asymptotic outage analysis of HARQ-IR over time-correlated Nakagami-m fading channels," IEEE Trans. Wireless Commun., vol. 16, no. 9, pp. 6119-6134, Sep. 2017.

[28] A. Chelli, E. Zedini, M.-S. Alouini, J. Barry, and M. Patzold, "Performance and delay analysis of hybrid ARQ with incremental redundancy over double Rayleigh fading channels," IEEE Trans. Wireless Commun., vol. 13, no. 11, pp. 6245-6258, Nov. 2014.

[29] F. Yilmaz and M.-S. Alouini, "Outage capacity of multicarrier systems," in Proc. IEEE Int. Conf. on Telecommunications (ICT'10), Apr. 2010, pp. 260-265.

[30] Q. Liu, S. Zhou, and G. B. Giannakis, "Cross-layer combining of adaptive modulation and coding with truncated ARQ over wireless links," IEEE Trans. Wireless Commun., vol. 3, no. 5, pp. 1746-1755, Sep. 2004.

[31] L. Debnath and D. Bhatta, Integral transforms and their applications. CRC press, 2010.

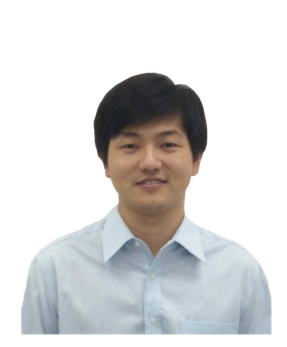

Zheng Shi received his B.S. degree in communication engineering from Anhui Normal University, China, in 2010 and his M.S. degree in communication and information system from Nanjing University of Posts and Telecommunications (NUPT), China, in 2013. He obtained his Ph.D. degree in Electrical and Computer Engineering from University of Macau, Macao, in 2017. Since 2017, he has been with the School of Intelligent Systems Science and Engineering, Jinan University and is now an assistant professor there. His research interests include hybrid automatic repeat request (HARQ) protocols, cooperative communications, full-duplex communications, non-orthogonal multiple access, millimeter wave and heterogeneous wireless networks.

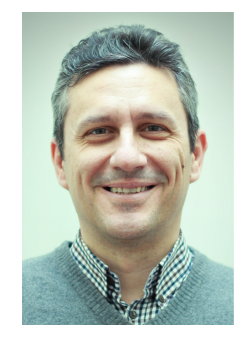

Theodoros A. Tsiftsis (S'02, M'04, SM'10) was born in Lamia, Greece, in 1970. He received the B.Sc. degree in physics from the Aristotle University of Thessaloniki, Greece, in 1993, the M.Sc. degree in digital systems engineering from the Heriot-Watt University, Edinburgh, U.K., in 1995, the M.Sc. degree in decision sciences from the Athens University of Economics and Business, in 2000, and the Ph.D. degree in electrical engineering from the University of Patras, Greece, in 2006. He is currently a Professor in the School of Intelligent Systems Science \& Engineering at Jinan University, Zhuhai Campus, Zhuhai, China. $\mathrm{He}$ has authored or co-authored over 180 technical papers in scientific journals and international conferences. His research interests include the broad areas of cognitive radio, communication theory, wireless powered communication systems, optical wireless communication, and ultra-reliable low-latency communication.

Prof. Tsiftsis has been appointed to a 2-year term as an IEEE Vehicular Technology Society Distinguished Lecturer (IEEE VTS DL), Class 2018. Dr. Tsiftsis acts as a Reviewer for several international journals and conferences. He has served as Senior or Associate Editor in the Editorial Boards of IEEE TRANSACTIONS ON VeHICULAR TECHNOLOGY, IEEE COMMUNICATIONS LETTERS, IET COMMUNICATIONS, and IEICE TRANSACTIONS ON COMMUNICATIONS. He is currently an Area Editor for Wireless Communications II of the IEEE TRANSACTIONS ON COMMUNICATIONS and an Associate Editor of the IEEE TRANSACTIONS ON MOBILE COMPUTING.

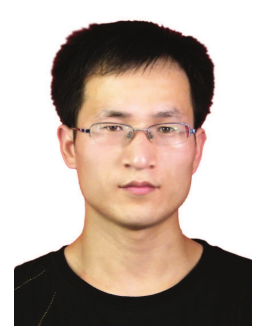

Weiqiang Tan received his $\mathrm{Ph} . \mathrm{D}$. degree from the National Mobile Communications Research Laboratory, Southeast University, Nanjing, China, in 2017, and his M.S. degree from Chengdu University of Information Technology, China, in 2013. From 2016 to 2017, he was a visiting Ph.D. student with the School of Electronics, Electrical Engineering and Computer Science, Queen's University Belfast, United Kingdom. He is currently with the School of Computer Science and Cyber Engineering, Guangzhou University, Guangzhou, 510006, P. R. China. His research interests include massive MIMO system and Millimeter wave wireless communication.

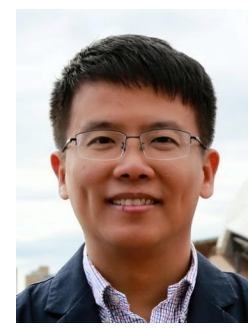

Guanghua Yang (S'02, M'07, SM'19) received his $\mathrm{Ph} . \mathrm{D}$. degree in electrical and electronic engineering from the University of Hong Kong, Hong Kong, in 2006. From 2006 to 2013, he served as post-doctoral fellow, research associate, and project manager in the University of Hong Kong. Since April 2017, he is an Associate Professor and Associate Dean with the Institute of Physical Internet, Jinan University, Guangdong, China. His research interests are in the general areas of communications, networking and multimedia. 


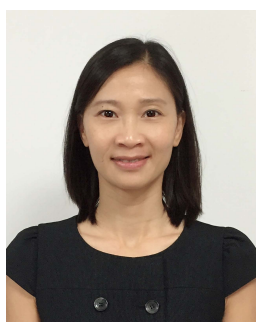

Shaodan Ma received her double Bachelor degrees in Science and Economics, and her Master degree of Engineering, from Nankai University, Tianjin, China. She obtained her Ph. D. degree in electrical and electronic engineering from the University of Hong Kong, Hong Kong, in 2006. From 2006 to 2011, she was a Postdoctoral Fellow in the University of Hong Kong. Since August 2011, she has been with the University of Macau and is now an Associate Professor there. She was a visiting scholar in Princeton University in 2010 and is currently an Honorary Assistant Professor in the University of Hong Kong. Her research interests are in the general areas of signal processing and communications, particularly, transceiver design, resource allocation and performance analysis.

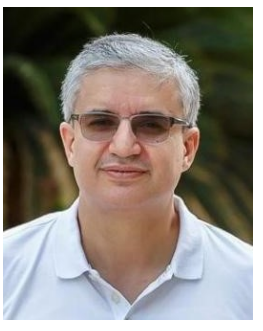

Mohamed-Slim Alouini (S'94-M'98-SM'03-F'09) was born in Tunis, Tunisia. He received the Ph.D. degree in Electrical Engineering from the California Institute of Technology (Caltech), Pasadena, CA, USA, in 1998. He served as a faculty member in the University of Minnesota, Minneapolis, MN, USA, then in the Texas A\&M University at Qatar, Education City, Doha, Qatar before joining King Abdullah University of Science and Technology (KAUST), Thuwal, Makkah Province, Saudi Arabia as a Professor of Electrical Engineering in 2009. His current research interests include the modeling, design, and performance analysis of wireless communication systems. 